\title{
A pseudospectral tau approximation for time delay systems and its comparison with other weighted-residual-type methods
}

\author{
David Lehotzky*, a and Tamas Insperger ${ }^{\mathrm{a}}$ \\ ${ }^{a}$ Department of Applied Mechanics, Budapest University of Technology and Economics, Hungary, \\ 1111, Budapest, Muegyetem rkp. 3.
}

\begin{abstract}
This paper presents a method which applies pseudospectral tau approximation for retarded functional differential equations (RFDEs). The goal is to construct a system of ordinary differential equations (ODEs), which provides a finite dimensional approximation of the original RFDE. The method can be used to determine approximate stability diagrams for RFDEs. Thorough numerical case studies show that the rightmost characteristic roots of the ODE approximation converge to the rightmost characteristic roots of the original RFDE. Application of the method to time-periodic RFDEs is also demonstrated, and the convergence of the stability boundaries is verified numerically. The method is compared with recently developed highly efficient numerical methods: the pseudospectral collocation (also called Chebyshev spectral continuous-time approximation), the spectral Legendre tau method and the spectral element method. The comparison is based on the stability analysis of three linear autonomous RFDEs. The efficiency of the methods is measured by the convergence rate of stability boundaries in the space of system parameters, by the convergence rate of the rightmost characteristic exponent and by the computation time of the stability charts.
\end{abstract}

Keywords: time delay; linear stability; numerical method; weighted residual method; tau method

${ }^{*}$ Corresponding author

E-mail address: lehotzky@mm.bme.hu 


\section{Introduction}

In the recent decades an increasing number of research papers and books have dealt with time-delay systems in applied sciences. Time delay emerged first in population dynamics but since then its significance has been discovered in many engineering and biological applications. For instance, control systems always involve feedback delays due to finite-time information transmission, signal processing and actuation $[1,2]$. Similarly, the nervous system of humans is subjected to delay $[3,4,5]$, which affects balancing abilities and may cause movement disorders. Reflex delay of the human nervous system is the main reason for the development of stop-and-go traffic jams [6, 7]. Time delay also plays an important role in contact problems such as the "shimmy motion" of wheels [8]. Machine tool vibrations are also explained by the so-called regenerative delay (for details, see Chapter 4.4 in [9] or $[10,11])$. In the above examples, time delay typically has a destabilizing effect, which is manifested as unwanted vibrations or oscillations around the desired steady-state motion. Stability analysis of the steady-state motions is therefore of high importance. Consequently, several numerical methods can be found in the literature for the stability analysis of linear time-delay systems.

This paper presents a pseudospectral tau (PsT) method for the approximation of retarded functional differential equations (RFDEs) by a system of ordinary differential equations (ODEs). Focusing on the stability of the original RFDE, some properties of the PsT method are analyzed through numerical case studies. Furthermore, the PsT method is compared with the pseudospectral collocation [12, 13] (PsC) also called Chebyshev spectral continuous-time approximation, with the spectral Legendre tau (SLT) method [14] and with the spectral element (SE) method [15]. The comparison is based on the stability analysis of three linear autonomous RFDEs: the Hayes equation, an oscillator with two delays and an oscillator with distributed delay. The stability properties of these systems can be determined in closed form, which serves as a reference for the numerical results. The efficiency of different methods is measured by the convergence rate of stability boundaries in the space of system parameters, by the convergence rate of the rightmost characteristic exponent and by the computation time of stability charts. Note that the methods under comparison all apply for the stability analysis of linear time-periodic systems as well.

In addition to the results on autonomous RFDEs, this paper also presents the application of the PsT method for the stability analysis of linear time-periodic RFDEs. The approximation concept is illustrated on two examples: the delayed Mathieu equation and an oscillator with time-periodic delay. The structure of this paper is the following. First, a brief summary is given on different forms of the equations under study. Then, the formulation of the PsT method is detailed for RFDEs of general type. Thereafter, comparison of the PsT method with the PsC, SE and SLT methods is presented. Then, the application of the PsT method is demonstrated for the stability analysis of time-periodic RFDEs on two examples. Finally, the paper is concluded in the last section.

\section{Equivalent forms of delayed problems}

The numerical methods (PsT, PsC, SLT, SE) discussed in this paper were derived for different representations of time-delay systems. In this section, these representations are detailed.

\subsection{Retarded functional differential equation}

The investigated linear RFDE has the form

$$
\dot{\mathbf{x}}(t)=\mathcal{L}(t) \mathbf{x}_{t}, \quad t \geq 0,
$$

where the dot represents the right-hand derivative with respect to time $t$ and $\mathcal{L}(t): \mathbb{R}^{+} \times \mathcal{X} \rightarrow \mathbb{R}^{s}$ is a continuous linear functional, with $\mathcal{X}=\mathcal{H}\left([-\tau, 0] ; \mathbb{R}^{s}\right)$ being the Hilbert space of continuous $\mathbb{R}^{s}$-valued functions on interval $[-\tau, 0]$. Note that $\mathcal{L}(t)$ depends on system parameters. In (1), function segment $\mathbf{x}_{t} \in \mathcal{X}$ is defined by the shift

$$
\mathbf{x}_{t}(\theta)=\mathbf{x}(t+\theta), \quad \theta \in[-\tau, 0] .
$$


It is clear from (1) that the evolution of an RFDE is determined from the solution segment $\mathbf{x}_{t}$ which contains the "history" of the state $\mathbf{x}$ dating back to time instant $t-\tau$. RFDEs are therefore infinite dimensional systems, and a solution operator $\mathcal{U}(t)$ maps the initial solution segment $\boldsymbol{x}_{0}$ to the solution segment $\boldsymbol{x}_{t}$. According to the Floquet theory, if $\mathcal{L}(t)$ is periodic (that is $\mathcal{L}(t)=\mathcal{L}(t+T)$ for all $t$ with $T$ being the principal period), then the stability of (1) is determined by the monodromy operator $\mathcal{U}(T)$. The nonzero eigenvalues of $\mathcal{U}(T)$ are called charactersitic mutlipliers and system (1) is asymptotically stable if and only if all the characteristic multipliers are within the unit circle of the complex plane (for details see Chapter 8 in [16]). If $\mathcal{L}$ is time-invariant, then the stability properties of (1) are determined by its characteristic roots (or characteristic exponents). The system is asymptotically stable if and only if the real part of the rightmost characteristic exponent is negative. Note that for time-invariant systems the principal period $T$ is not defined but the Floquet theory can still be applied with an arbitrary principal period.

\subsection{Operator equation}

Let operator $\mathcal{V}: \mathcal{D}(\mathcal{V}) \rightarrow \mathcal{X}^{ \pm}$be defined as

$$
\mathcal{V}\left(\mathbf{x}_{0}, \mathbf{x}^{+}\right)(\varphi)= \begin{cases}\mathbf{x}_{0}(\varphi) & \text { if } \varphi \in[-\tau, 0] \\ \mathbf{x}^{+}(\varphi) & \text { if } \varphi \in(0, h]\end{cases}
$$

with domain

$$
\mathcal{D}(\mathcal{V})=\left\{\mathbf{x}_{0} \in \mathcal{X}, \mathbf{x}^{+} \in \mathcal{X}^{+}: \mathbf{x}_{0}(0)=\mathbf{x}^{+}(0)\right\} .
$$

Here $h>0$ and $\mathcal{X}^{ \pm} \subset \mathcal{H}\left([-\tau, h], \mathbb{R}^{s}\right)$, while $\mathcal{X}^{+}=\mathcal{H}^{1}\left([0, h], \mathbb{R}^{s}\right)$ is the Hilbert space of $\mathbb{R}^{s}$-valued continuously differentiable functions on interval $[0, h]$. Note that operator $\mathcal{V}\left(\mathbf{x}_{0}, \mathbf{x}^{+}\right)$simply connects the initial function segment $\mathbf{x}_{0}$ and function segment $\mathbf{x}^{+}$at $\varphi=0$. Now considering the residual of (1) on $t \in[0, h]$, one can construct the operator equation (OpE)

$$
\mathcal{A} \mathbf{z}=\mathbf{0},
$$

where operator $\mathcal{A}: \mathcal{X}^{ \pm} \rightarrow \mathcal{H}\left([0, h], \mathbb{R}^{s}\right)$ is defined by

$$
\mathcal{A} \mathbf{z}=\left\{\dot{\mathbf{z}}(t)-\mathcal{L}(t) \mathbf{z}_{t}: t \in[0, h]\right\} .
$$

Since $\mathcal{L}(t)$ is linear, one can decompose (5) by plugging (3) into (5) as

$$
\mathcal{A}^{-} \mathbf{x}_{0}+\mathcal{A}^{+} \mathbf{x}^{+}=\mathbf{0}
$$

where

$$
\mathcal{A}^{-} \mathrm{x}_{0}=\mathcal{A} \mathcal{V}\left(\mathrm{x}_{0}, \mathbf{0}\right), \quad \mathcal{A}^{+} \mathrm{x}^{+}=\mathcal{A} \mathcal{V}\left(\mathbf{0}, \mathrm{x}^{+}\right)
$$

For any $\mathbf{x}_{0} \in \mathcal{X}$ initial function segment, the solution of (7) is precisely defined by $\mathbf{x}^{+}=\{\mathbf{x}(t): t \in[0, h]\}$. When $\mathcal{L}(t)$ is time-periodic and $h=T$, the monodromy operator $\mathcal{U}(T)$ can be expressed using $(7)$ (see Section 5.3.3). Therefore, (7) can be used for the stability analysis of (1).

\subsection{Operator differential equation}

Stability properties of (1) can be described by the operator differential equation (OpDE)

$$
\dot{\mathbf{x}}_{t}=\mathcal{G}(t) \mathbf{x}_{t}, \quad t>0,
$$

where operator $\mathcal{G}: \mathcal{D}(\mathcal{G}) \rightarrow \mathcal{X}$ is given by

$$
\mathcal{G}(t) \mathbf{x}_{t}=\mathbf{x}_{t}^{\prime},
$$

with domain $\mathcal{D}(\mathcal{G})=\mathbb{R}^{+} \times \mathcal{Y}$, where

$$
\mathcal{Y}=\left\{\mathbf{x}_{t} \in \mathcal{X}: \mathbf{x}_{t}^{\prime} \in \mathcal{X}, \mathbf{x}_{t}^{\prime}(0)=\mathcal{L}(t) \mathbf{x}_{t}\right\} \subseteq \mathcal{X}
$$


and $\mathbf{x}_{t}^{\prime}(\theta)$ is the derivative of $\mathbf{x}_{t}(\theta)$ with respect to $\theta$. Note that $\mathcal{G}$ cannot be correctly defined when $\mathcal{Y}$ is time-dependent, hence varying (see page 341 of [17]). Therefore, here we assume that $\mathcal{Y}$ can be selected as a time-independent domain (e.g., the maximum delay is finite). When $\mathcal{G}$ is time-invariant then (9) gives an abstract Cauchy problem and operator $\mathcal{G}$ is called its infinitesimal generator. If and only if all the elements of the spectrum of the infinitesimal generator lie on the left half of the complex plane, then the abstract Cauchy problem is asymptotically stable.

In Chapter 7.1 of [16], Lemma 1.2. shows that the solution of the abstract Cauchy problem is defined by the solution operator $\mathcal{U}(t)$. Furthermore, in Chapter 7.2 of [16], it is also shown that the spectrum of $\mathcal{G}$ is precisely given by the roots of the characteristic equation of (1). This latter implies that, for the autonomous case, the stability analysis of (1) and (9) gives the same results. Numerical results presented in [13] showed that (1) and (9) are equivalent regarding stability for time-periodic systems as well. Note, that the conversion of (1) to (9) is similar to the conversion of a high-order ODE to first-order ODEs (Cauchy normal form).

By the introduction of function $\mathbf{y}(t, \theta)=\mathbf{x}_{t}(\theta)$ with two independent variables $t$ and $\theta$, (9) gives the hyperbolic PDE

$$
\frac{\partial \mathbf{y}(t, \theta)}{\partial t}=\frac{\partial \mathbf{y}(t, \theta)}{\partial \theta}, \quad \theta \in[-\tau, 0], \quad t>0
$$

with the linear, time-dependent, non-local boundary condition

$$
\left.\frac{\partial \mathbf{y}(t, \theta)}{\partial t}\right|_{\theta=0}=\mathcal{L}(t) \mathbf{y}(t, \theta), \quad t>0
$$

This PDE representation is equivalent to (9) and it is often used in the literature to describe delayed systems $[14,18]$. Note, that in some engineering applications the governing equation which models the physical phenomenon can be derived directly in the form (12)-(13), instead of (1). For example, the widely used RFDE model of turning with constant delay (see Chapter 5.1.2 in [19]) is a special case of the PDE model, introduced in [20]. In particular, the RFDE model describes the cutting tool's motion in the PDE model under the condition that the tool never loses contact with the workpiece (for details see [21]). In general, equations (12)-(13) are capable of giving a more detailed description of the delayed system than equation (1). This is due to the fact that in the PDE model, (12) describes the propagation of information while (13) introduces delays in the system. In contrast, RFDE (1) embeds the propagation in the time domain using time lags. During mathematical modelling, a decision between the use of (12)-(13) or (1) always involves a trade-off since, in general, the analysis of PDEs is more difficult than the analysis of RFDEs.

\section{Method of weighted residuals}

The numerical methods which are compared in this article use the method of weighted residuals. In this section, this method is detailed for (9).

At time instant $t$, one can approximate the solution segment $\mathbf{x}_{t}(\theta)$ of $(9)$ on the domain $\theta \in[-\tau, 0]$, using finite number of unknown variables $\mathbf{a}_{j}(t)$ in a finite dimensional function space spanned by the basis $\left\{\phi_{j}\right\}_{j=1}^{n}$. The approximate solution of (9) therefore has the form

$$
\tilde{\mathbf{x}}_{t}(\theta)=\sum_{j=1}^{n} \mathbf{a}_{j}(t) \phi_{j}(\theta)
$$

After the substitution of $\tilde{\mathbf{x}}_{t}$ into (9), one obtains the residual function

$$
\mathbf{r}_{t}(\theta)=\sum_{j=1}^{n} \dot{\mathbf{a}}_{j}(t) \phi_{j}(\theta)-\sum_{j=1}^{n} \mathbf{a}_{j}(t) \phi_{j}^{\prime}(\theta) \neq \mathbf{0}, \quad \theta \in[-\tau, 0] .
$$

Note that in general the residual function is not zero since $\tilde{\mathbf{x}}_{t}$ is only an approximation of $\mathbf{x}_{t}$. Approximation schemes aim to determine coefficients $\mathbf{a}_{j}(t)$ in a way that the approximate solution segment $\tilde{\mathbf{x}}_{t}$ 
be closest to the exact solution of (9). The method of weighted residuals weighs the residual function $\mathbf{r}_{t}(\theta)$ by test functions $\psi_{i}(\theta)$ over the domain $\theta \in[-\tau, 0]$ in order to obtain a set of linearly independent equations, from which coefficients $\mathbf{a}_{j}(t)$ can be determined. The application of the method of weighted residuals to (15), gives

$$
\left\langle\mathbf{r}_{t}, \psi_{i}\right\rangle=0, \quad i=1,2, \ldots, n ;
$$

where the inner product of functions $f(\theta)$ and $g(\theta)$ is defined according to

$$
\langle f, g\rangle=\int_{a}^{b} f(\theta) g(\theta) \mathrm{d} \theta
$$

with $\theta \in[a, b]$ being the domain of functions $f(\theta)$ and $g(\theta)$. Equations (16) can be represented in a matrix form as

$$
\mathbf{N a}(t)=\mathbf{M a}(t),
$$

where matrices $\mathbf{N} \in \mathbb{R}^{s n \times s n}$ and $\mathbf{M} \in \mathbb{R}^{s n \times s n}$ are composed from sub-matrices according to

$$
\mathbf{N}=\left[\left\langle\phi_{j}, \psi_{i}\right\rangle \mathbf{I}\right]_{i, j=1}^{n, n}, \quad \mathbf{M}=\left[\left\langle\phi_{j}^{\prime}, \psi_{i}\right\rangle \mathbf{I}\right]_{i, j=1}^{n, n},
$$

while $\mathbf{I} \in \mathbb{R}^{s \times s}$ is an identity matrix and $\mathbf{a}(t)=\left[\mathbf{a}_{j}(t)\right]_{j=1}^{n}$. Note that due to the application of the method of weighted residuals the state space $\mathcal{X}$ has to be a Hilbert space, however the proper selection of $\mathcal{X}$ falls out of the scope of this paper. Note also that the solution of (16) is not in the domain $\mathcal{D}(\mathcal{G})$ since boundary condition

$$
\tilde{\mathbf{x}}_{t}^{\prime}(0)=\mathcal{L}(t) \tilde{\mathbf{x}}_{t}
$$

is not satisfied. For the PDE representation this means that (12) is employed but (13) is not satisfied. Consequently, in order to approximate (9), boundary condition (20) has to be enforced.

Based on the above description, weighted residual type methods can be different from each other in the way they select the set of base functions and the set of test functions and also in the way they enforce the boundary conditions. Methods can be categorized based on how they enforce the boundary conditions. There are two main categories which are briefly discussed below.

\subsection{Galerkin approximation}

For the Galerkin method the boundary conditions are considered as constraints on the approximate solution $\tilde{\mathbf{x}}_{t}$, that is, base functions $\phi_{j}$ are constructed in a way that $\tilde{\mathbf{x}}_{t}$ satisfies the boundary condition. Note, however that boundary condition (20) is non-local, that is, it requires the exact solution of (1) to be known. Thus, the non-locality of boundary condition (20) implies that Galerkin methods cannot be used for the approximation of (9).

\subsection{Tau approximation}

In order to solve the problem with non-local boundary conditions, the tau approximation can be used. In contrast to the Galerkin method, base functions $\phi_{j}$ do not need to satisfy the boundary constraints. The tau approximation technique simply replaces an equation from (16) by the discretized boundary condition (20). This replacement relies on the tau method which was proposed by Lanczos (see [22]). The tau method claims that if, after this replacement, (16) still defines a proper projection then the approximate solution is an element of a complete finite dimensional subspace of the solutions of the original problem (9). Note again, that in this case $\mathcal{X}$ has to be a proper Hilbert space. More precise details on Galerkin and tau approximations can be found in Chapter 2 of [23].

\section{Pseudospectral tau approximation}

For the PsT method, the term "pseudospectral" indicates that the solution is approximated in a finite dimensional subspace, where the set of basis functions $\left\{\phi_{j}\right\}_{j=1}^{n}$ are chosen in a way that the coordinates $\mathbf{a}_{j}$ of the subspace spanned by $\left\{\phi_{j}\right\}_{j=1}^{n}$ represent the approximate solution at specific points. In the following, the approximation concept of the PsT method is given in details. 


\subsection{Lagrange interpolation}

The PsT method approximates the solution segment by its Lagrange interpolant. The approximate solution segment is given in the form

$$
\tilde{\mathbf{x}}_{t}(\theta)=\sum_{j=1}^{n} \phi_{j}(\theta) \mathbf{x}_{t}\left(\theta_{j}\right), \quad \theta \in[-\tau, 0],
$$

where $\theta_{j} \in[-\tau, 0]$ are the nodes of interpolation and $\phi_{j} \in \mathcal{P}_{n-1}$ are the Lagrange base polynomials, with $\mathcal{P}_{n-1}$ denoting the space of polynomials of order $n-1$. Note that by using interpolant (21), the unknown coefficients of (14) become particular values of the approximate solution segment at some points $\theta_{j}$, that is $\mathbf{a}_{j}(t)=\mathbf{x}_{t}\left(\theta_{j}\right)$. Lagrange base polynomials have the property

$$
\phi_{j}\left(\theta_{k}\right)=\delta_{j, k},
$$

where $\delta_{j, k}$ denotes the Kronecker-delta function. The classical form of Lagrange base polynomials is

$$
\phi_{j}(\theta)=\prod_{\substack{k=1 \\ k \neq j}}^{n} \frac{\theta-\theta_{k}}{\theta_{j}-\theta_{k}} .
$$

However, the above formula has some disadvantages: it needs high number of floating point operations to calculate $\phi_{j}$ at any given point other than the nodes of interpolation, while the rounding errors can lead to numerical instability, furthermore the formula for the derivative of $\phi_{j}$ is very complicated. The so-called barycentric representation of Lagrange interpolants helps in the above problems. The barycentric formula of Lagrange base polynomials is defined by

$$
\phi_{j}(\theta)=\frac{\frac{\varpi_{j}}{\theta-\theta_{j}}}{\sum_{k=1}^{n} \frac{\varpi_{k}}{\theta-\theta_{k}}},
$$

where the barycentric weights are given as

$$
\varpi_{j}=\frac{1}{\omega^{\prime}\left(\theta_{j}\right)}, \quad \omega(\theta)=\prod_{j=1}^{n}\left(\theta-\theta_{j}\right) .
$$

At the nodes of interpolation the derivatives of the Lagrange base polynomials can be calculated as

$$
\phi_{j}^{\prime}\left(\theta_{k}\right)= \begin{cases}\frac{\varpi_{j} / \varpi_{k}}{\theta_{k}-\theta_{j}} & j \neq k, \\ -\sum_{\substack{q=1 \\ q \neq j}}^{n} \frac{\varpi_{q} / \varpi_{j}}{\theta_{j}-\theta_{q}} & j=k .\end{cases}
$$

Details and derivation of the above formulae can be found in [24]. Note, that using (26), the derivative of the Lagrange interpolant at the nodes of interpolation can be calculated simply by a matrix-vector multiplication, since

$$
\tilde{\mathbf{x}}_{t}^{\prime}\left(\theta_{j}\right)=\sum_{j=1}^{n} D_{k, j} \tilde{\mathbf{x}}_{t}\left(\theta_{j}\right), \quad k=1, \ldots, n ;
$$

where $D_{k, j}=\phi_{j}^{\prime}\left(\theta_{k}\right)$. Note that the value of the Lagrange interpolant at $n$ number of arbitrary distinct points $\left\{\theta_{l}^{*}\right\}_{l=1}^{n}$ is given by a matrix-vector multiplication:

$$
\tilde{\mathbf{x}}_{t}\left(\theta_{l}^{*}\right)=\sum_{j=1}^{n} L_{l, j} \tilde{\mathbf{x}}_{t}\left(\theta_{j}\right), \quad l=1, \ldots, n ;
$$

where $L_{l, j}=\phi_{j}\left(\theta_{l}^{*}\right)$ defines a linear transformation between the two point sets defined by the interpolant evaluated at node sets $\left\{\theta_{l}^{*}\right\}_{l=1}^{n}$ and $\left\{\theta_{j}\right\}_{j=1}^{n}$. Due to the uniqueness of the Lagrange interpolant, its derivative can be calculated at any given point set $\left\{\theta_{l}^{*}\right\}_{l=1}^{n}$ by the matrix-matrix-vector multiplication

$$
\tilde{\mathbf{x}}_{t}^{\prime}\left(\theta_{l}^{*}\right)=\sum_{k=1}^{n} \sum_{j=1}^{n} L_{l, k} D_{k, j} \tilde{\mathbf{x}}_{t}\left(\theta_{j}\right), \quad l=1, \ldots, n .
$$




\subsection{Tau approximation}

Using (21), after the replacement of the equation corresponding to $i=n$ in (16) with the discretized boundary condition (20) and coordinate transformation $\zeta=2 \theta / \tau+1$, equation (18) obtains the form

$$
\mathbf{N} \dot{\mathbf{X}}(t)=\mathbf{M}(t) \mathbf{X}(t),
$$

where $\mathbf{X}(t)=\left[\tilde{\mathbf{x}}_{t}\left(\theta_{j}\right)\right]_{j=1}^{n}$ while the elements of matrices $\mathbf{N}$ and $\mathbf{M}(t)$ are now given according to

$$
\begin{aligned}
\mathbf{N}_{i, j} & = \begin{cases}\left\langle\phi_{j}, \psi_{i}\right\rangle \mathbf{I} & i=1, \ldots, n-1 ; \\
\phi_{j}(1) \mathbf{I} & i=n ;\end{cases} \\
\mathbf{M}_{i, j}(t) & = \begin{cases}\frac{2}{\tau}\left\langle\phi_{j}^{\prime}, \psi_{i}\right\rangle \mathbf{I} & i=1, \ldots, n-1 ; \\
\mathcal{L}(t) \phi_{j} & i=n .\end{cases}
\end{aligned}
$$

If $\mathbf{N}$ is invertible then (30) can be written as

$$
\dot{\mathbf{X}}(t)=\mathbf{G}(t) \mathbf{X}(t),
$$

where $\mathbf{G}(t)=\mathbf{N}^{-1} \mathbf{M}(t)$ is a finite dimensional approximation of operator $\mathcal{G}(t)$. Note that in (16) the multiplication of residual function (15) with test functions $\psi_{i}, i=1, \ldots, n-1$ gives the projection of the residual function onto a space spanned by the test function set $\left\{\psi_{i}\right\}_{i=1}^{n-1}$. Since the approximate solution (21) is an element of the subspace of polynomials of order $n-1$, by choosing the test function set as a base in the subspace of polynomials of order $n-2$, the residual (15) will be zero in a codimension one subspace of the space $\mathcal{P}_{n-1}$ of (21). The boundary condition is enforced by (20), which gives additional relationship between coefficients $\mathbf{x}_{t}\left(\theta_{j}\right)$. If a proper set of test functions is chosen, the solution of the system subject to tau approximation can be an element of a complete finite dimensional subspace of the solution of the original problem (9). Consequently, by increasing $n$ the approximation can converge to the original problem. In this paper, the suitability of the test function set is not investigated analytically, but they have been tested numerically. The numerical experiments showed that for the autonomous case the eigenvalues of $\mathbf{G}$ in (33) give a finite dimensional approximation for the point spectrum of $\mathcal{G}$.

Although this method is capable of the approximation of the point spectrum of $\mathcal{G}$, the main focus of this paper is only on the numerical stability analysis of RFDEs. Since the ODE approximation (33) is stable if and only if all eigenvalues of $\mathbf{G}$ are located in the left half of the complex plane, only the rightmost eigenvalue of $\mathbf{G}$ is of interest. Note that if RFDE (1) is modified that affects only the last rows of $\mathbf{M}(t)$ corresponding to $i=n$ and the multiplier of the remaining rows. Therefore, when $s$ and $n$ are fixed, the inverse of $\mathbf{N}$ and the integral terms in (31)-(32) have to be calculated only once, which is beneficial during the construction of stability charts (as discussed in Section 5.4)

In Figure 1, an illustration of the ODE approximation (33) is shown for $s=1$ and $n=3$. The solution of the ODE approximation is presented in Figures 1/A and 1/B together with the solution for the PDE (12)-(13) and OpDE (9) representations of RFDE (1), respectively. In Figure 1, the exact solution is shown by blue color while the solution of the ODE approximation is depicted by red color. The thin red lines are given by the points of the interpolant at the nodes of interpolation, that is they are given by the elements of $\mathbf{X}(t)$. In Figure 1/A, these lines define the red surface which approximates the exact solution of (12)-(13). Note that, due to (12), the isocurves of the blue surface are lines with slope 1. In Figure 1/B the thin red lines define an approximation for the solution segment $x_{t}$ of (9) at each time instant $t$. These solution segment approximations are shown by thick red lines for time instants $0, t_{1}$ and $t_{2}$. At each $t$ time instant, the solution segment $x_{t}$ coincides with the corresponding segment of $y(t, \theta)$. This is highlighted by gray windows in Figures $1 / \mathrm{A}$ and $1 / \mathrm{B}$ for time instants 0 , $t_{1}$ and $t_{2}$. Note that each element of $\mathbf{X}(t)$ gives an approximation for the solution $x(t)$ of RFDE (1) due to the definition (2) of solution segment $x_{t}$. This is illustrated in Figure 1/C.

\subsection{Numerical integration}

Equations (31)-(32) contain integral terms whose analytical evaluation would be time-consuming or even impossible. Note however, that since $\left\{\psi_{i}\right\}_{i=1}^{n-1} \subset \mathcal{P}_{n-2}$, the terms in the integral are polynomials 


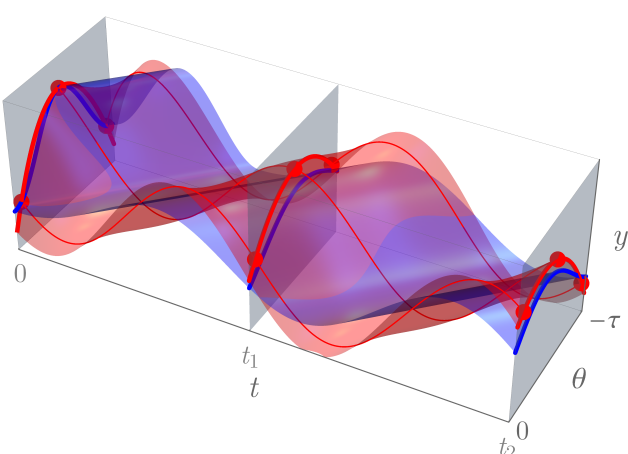

A)

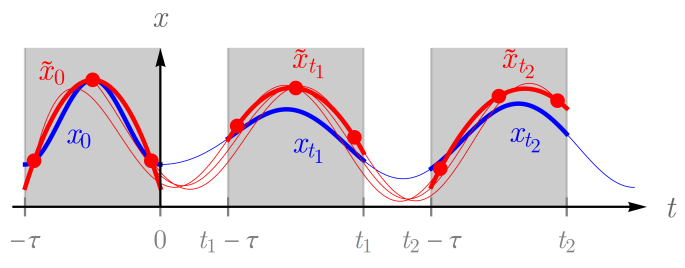

B)

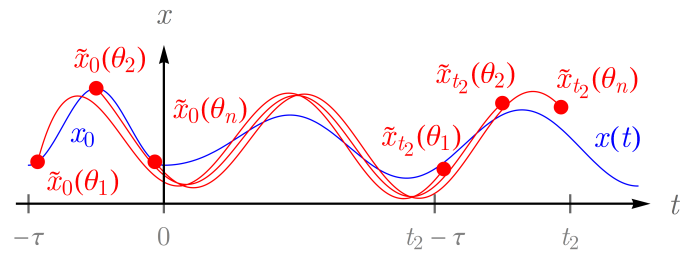

C)

Figure 1: Representation of the solution A) of PDE (12)-(13) (blue surface), B) of OpDE (9) (thick blue lines) and C) of RFDE (1) (blue line), and their ODE approximations (red surface and lines)

with maximum order $2 n-3$. As a result, the integral terms in (31)-(32) can be evaluated accurately by the Legendre-Gauss-Lobatto quadrature (see Appendix B for details) using $n$ number of points. With the application of this quadrature, the integral terms in (31)-(32) can be calculated as

$$
\begin{aligned}
\left\langle\phi_{j}, \psi_{i}\right\rangle & =\sum_{q=1}^{n} F_{i, q} L_{q, j}, \quad i=1, \ldots, n-1 ; \\
\left\langle\phi_{j}^{\prime}, \psi_{i}\right\rangle & =\sum_{q=1}^{n} \sum_{k=1}^{n} F_{i, q} L_{q, k} D_{k, j}, \quad i=1, \ldots, n-1 ;
\end{aligned}
$$

where $F_{i, q}=\psi_{i}\left(\zeta_{q}^{*}\right) w_{q}$ with $\left\{\zeta_{q}^{*}\right\}_{q=1}^{n}$ and $\left\{w_{q}\right\}_{q=1}^{n}$ being the set of nodes and the corresponding set of weights of the quadrature. Note that if the interpolation and quadrature node sets are the same, then $L_{q, j}=\delta_{q, j}$, that is formulas (34)-(35) are simplified by one matrix multiplication. Also note that with the application of the standard (non-Lobatto-type) Legendre-Gauss quadrature for integration, the number of multiplications could be further decreased. This is owing to the fact that this quadrature gives accurate results for the integral of polynomials of order $2 n-3$ by using only $n-1$ number of nodes.

\subsection{Example}

Consider the system of RFDEs

$$
\dot{\mathbf{x}}(t)=\mathbf{B}(t) \mathbf{x}(t)+\sum_{p=1}^{r} \mathbf{C}_{p}(t) \mathbf{x}\left(t-\tau_{p}(t)\right)+\int_{-a}^{-b} \gamma(t, \theta) \mathbf{x}(t+\theta) \mathrm{d} \theta,
$$

where $\mathbf{x}: \mathbb{R} \rightarrow \mathbb{R}^{s}, \mathbf{B}: \mathbb{R} \rightarrow \mathbb{R}^{s \times s}, \mathbf{C}_{p}: \mathbb{R} \rightarrow \mathbb{R}^{s \times s}$ and $\tau_{p}: \mathbb{R} \rightarrow \mathbb{R} \forall p, \gamma: \mathbb{R}^{2} \rightarrow \mathbb{R}^{s \times s}$ and $s, p, r \in \mathbb{N}$. Furthermore $a>b \geq 0$ and $\tau_{p}(t) \geq 0 \forall t, \forall p$ is assumed. The substitution of (34)-(35) into (31)-(32) gives

$$
\begin{aligned}
\mathbf{N}_{i, j} & = \begin{cases}\sum_{q=1}^{n} F_{i, q} L_{q, j} \mathbf{I} & i=1, \ldots, n-1 ; \\
\phi_{j}(1) \mathbf{I} & i=n ;\end{cases} \\
\mathbf{M}_{i, j}(t) & = \begin{cases}\frac{2}{\tau} \sum_{q=1}^{n} \sum_{k=1}^{n} F_{i, q} L_{q, k} D_{k, j} \mathbf{I} & i=1, \ldots, n-1 ; \\
\mathcal{L}(t) \phi_{j} & i=n ;\end{cases}
\end{aligned}
$$


where $\mathbf{N}_{i, j}$ and $\mathbf{M}_{i, j}(t) \in \mathbb{R}^{s \times s}$, while $\tau=\max \left(a, \max \left(\left\{\sup \tau_{p}(t)\right\}_{p=1}^{r}\right)\right)$. Using Legendre-GaussLobatto quadrature for integration the last row of $\mathbf{M}_{i, j}(t)$ can be expanded as

$$
\mathcal{L}(t) \phi_{j} \approx \mathbf{B}(t) \phi_{j}(1)+\sum_{p=1}^{r} \mathbf{C}_{p}(t) \phi_{j}\left(-\hat{\tau}_{p}(t)\right)+\frac{\tau(\hat{b}-\hat{a})}{4} \sum_{q=1}^{n} \gamma\left(t, \frac{\left(\hat{\zeta}_{q}-1\right) \tau}{2}\right) \phi_{j}\left(\hat{\zeta}_{q}\right) w_{q}
$$

where $\hat{a}=1-2 a / \tau, \hat{b}=1-2 b / \tau, \hat{\tau}_{p}=-1+2 \tau_{p} / \tau \forall p$ and $\hat{\zeta}_{q}=\frac{\hat{b}-\hat{a}}{2}\left(\zeta_{q}^{*}+1\right)+\hat{a} \forall q$, while Lagrange base polynomials $\phi_{j}(\zeta)$ are defined by the node set $\left\{\zeta_{j}\right\}_{j=1}^{n}$ on the rescaled domain $\zeta \in[-1,1]$. Finally, the finite dimensional approximation of $\mathcal{G}(t)$ is given by (30)-(33).

\subsection{Selection of node and test function sets}

Note that due to the Lagrange interpolation, the base function set $\left\{\phi_{j}\right\}_{j=1}^{n}$ is precisely defined by the node set $\left\{\zeta_{j}\right\}_{j=1}^{n}$ of interpolation. Consequently, the error between the interpolated function segment $\mathbf{x}_{t}$ and its interpolant $\tilde{\mathbf{x}}_{t}$ can be minimized by the proper selection of node set $\left\{\zeta_{j}\right\}_{j=1}^{n}$. The error $\left|\mathbf{x}_{t}(\zeta)-\tilde{\mathbf{x}}_{t}(\zeta)\right|_{C[-1,1]}$ between $\mathbf{x}_{t}$ and $\tilde{\mathbf{x}}_{t}$ can be minimized by the selection of Chebyshev nodes for the nodes of interpolation, where the distance between $\mathbf{x}_{t}$ and $\tilde{\mathbf{x}}_{t}$ is measured by the norm

$$
\left|\mathbf{x}_{t}(\theta)\right|_{C[a, b]}=\max \left\{\left|\mathbf{x}_{t}(\theta)\right|: \theta \in[a, b]\right\} .
$$

Details on Chebyshev nodes and polynomials can be found in Appendix C. Due to its minimum error property, the Chebyshev node set is used for the PsT method as the node set of interpolation.

For the test function set $\left\{\psi_{i}\right\}_{i=1}^{n-1}$ two choices were investigated: the set $\left\{\zeta^{i-1}\right\}_{i=1}^{n-1}$ (which is a base in $\mathcal{P}_{n-2}$ ), and the set defined by Legendre polynomials of order up to $n-2$ (which is an orthogonal base in $\mathcal{P}_{n-2}$ ). For details on Legendre polynomials see Appendix A. In case of low $n$, these sets give precisely the same results for the eigenvalues of $\mathbf{G}$. However, above a certain order, the set $\left\{\zeta^{i-1}\right\}_{i=1}^{n-1}$ results in badly conditioned matrix $\mathbf{N}$, which destroys the convergence of the method. This problem is avoided, by using an orthogonal base in $\mathcal{P}_{n-2}$ (e.g. the Legendre polynomials). Consequently, for the PsT method, the test functions are defined as $\psi_{i}=P_{i-1}, i=1, \ldots, n-1$; where $P_{i}$ is a Legendre polynomial of order $i$.

\section{Comparison}

In this section the efficiency of the PsT method is compared with three methods from the literature. Comparison is made based on results, obtained for three linear autonomous RFDEs: the Hayes equation, an oscillator with two delays and an oscillator with distributed delay. In order to provide a base for comparison it is necessary to calculate the exact stability boundaries and rightmost characteristic exponents of the investigated equations. These are detailed in the sequel.

\subsection{Exact stability boundaries}

For the determination of stability boundaries the D-subdivision method [9] is used which utilizes the fact that when a root is crossing the stability boundary then its real part is zero, thus it has the form $\lambda=\mathrm{i} \beta$. Substituting this into the characteristic equation of (1) one can determine co-dimension 1 surfaces in the space of system parameters with running parameter $\beta$. When there are only two system parameters these co-dimension 1 surfaces result in the so-called D-curves on the plane of system parameters. These D-curves split the plane of system parameters onto domains where the number of unstable characteristic roots is the same. There are different methods to find the domains with zero unstable roots (i.e., the stable regions). If the number of unstable characteristic roots is known in one domain bounded by the D-curves, then the stable domains can be traced back using the concept of root crossing direction (see Chapter 3.4 in [25]). The number of unstable characteristic roots can also be calculated using Stepan's formulas (see Theorem 2.19. in [9]). 


\subsubsection{Hayes equation}

The Hayes equation reads

$$
\dot{x}(t)=a x(t)+b x(t-\tau) .
$$

The D-curves are defined by the parametric curves

$$
\begin{aligned}
& \beta=0: b=-a, \\
& \beta \tau \neq k \pi, k \in \mathbb{N}: \quad a=\frac{\beta \cos (\beta \tau)}{\sin (\beta \tau)}, b=\frac{-\beta}{\sin (\beta \tau)}
\end{aligned}
$$

in the parameter plane $(a, b)$ with the running parameter $\beta \in[0, \infty)$ (for details, see Chapter 2.1.1 of [19]). For this equation, the case $\tau=1$ is analysed throughout this paper. The D-curves and the domain of stability are shown in Figure 2/A. The D-curves are depicted with gray and black colors, and the stable domains are indicated with gray shading and black borders.

\subsubsection{Oscillator with two delays}

This RFDE has the form

$$
\ddot{x}(t)+a x(t)=b x\left(t-\tau_{1}\right)+b x\left(t-\tau_{2}\right),
$$

where $a>0$ is assumed. The D-curves are given by equations

$$
\begin{array}{r}
-\left(\frac{2 k \pi}{\tau_{1}+\tau_{2}}\right)^{2}+a-2 b(-1)^{k} \cos \left(k \pi \frac{\tau_{1}-\tau_{2}}{\tau_{1}+\tau_{2}}\right)=0, \quad k \in \mathbb{Z}, \\
\tau_{2}-\tau_{1}-\frac{2 k+1}{\sqrt{a}} \pi=0, \quad k \in \mathbb{Z} .
\end{array}
$$

Details on derivation can be found at the end of Chapter 3.1 in [9]. Throughout this paper, parameters $a=6$ and $b=1$ are used. The D-curves and the domain of stability are shown in Figure 2/B.

\subsubsection{Oscillator with distributed delay}

The general form of this RFDE is given by

$$
\ddot{x}(t)+a x(t)=b \int_{-\tau}^{0} \gamma(\theta) x(t+\theta) \mathrm{d} \theta,
$$

where the weight function and the length of time history are chosen as $\gamma(\theta)=\pi \sin (\pi \theta) / 2$ and $\tau=1$, respectively. The D-curves are given by

$$
\begin{aligned}
& a=(k \pi)^{2}-\frac{1+(-1)^{k}}{2\left(1-k^{2}\right)} b, \quad k \in \mathbb{Z} \backslash\{-1,1\}, \\
& b=0, \quad k= \pm 1 .
\end{aligned}
$$

Proof and detailed description on the derivation of stable parameter domains can be found in Theorem 3.26 in [9]. The D-curves and the domain of stability are shown in Figure 2/C.

\subsection{Exact value of the rightmost root}

Throughout this paper, the exact value of the rightmost characteristic root in any given point of the space of system parameters is determined using the corresponding characteristic equation. After the substitution of $\lambda=\alpha+\mathrm{i} \beta$ for the characteristic root, $\alpha$ and $\beta$ can be determined by solving the 


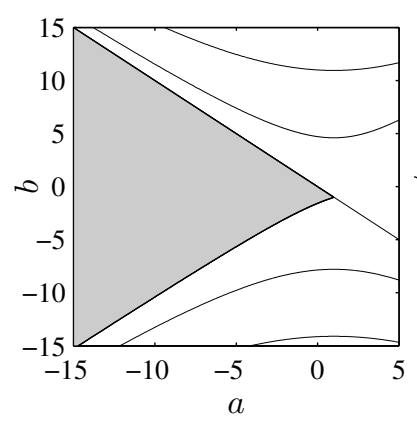

A)

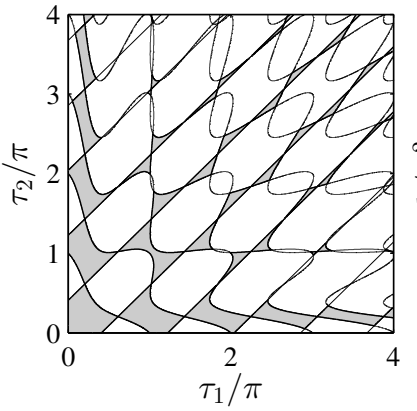

B)

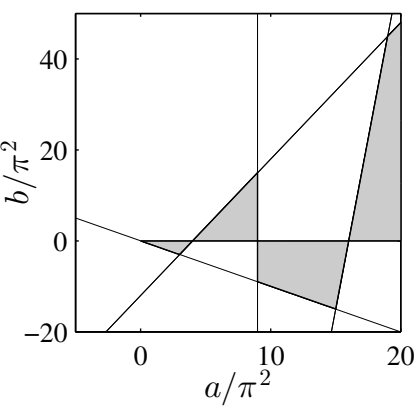

C)

Figure 2: Exact stability charts with D-curves: A) Hayes equation, B) Oscillator with two delays, C) Oscillator with distributed delay

system of non-linear equations given by the real and imaginary parts of the characteristic equation. However, in general, a solution of this system of equations can be found by using some iterative numerical method. In order to calculate the rightmost root, one should have a close enough initial guess. Here, and in the sequel, this initial guess is produced by the results of the above presented PsT method. In particular, the root used as initial guess, is the rightmost root taken from the results of the PsT method after the relative error converged to a value close to machine precision. The numerical solver used for the non-linear characteristic equation is the built-in solver of the software Wolfram Mathematica 9. The Newton-Rhapson method was used, such that the precision goal was set to be the double of the precision of the solution based on the PsT method. It has been experienced that starting the iteration from the rightmost root produced by the PsT method, the numerical solution of the characteristic equation gives practically the same result for all the investigated points in the space of system parameters.

\subsection{Methods under comparison}

In this subsection the different methods under comparison are described in detail.

\subsubsection{Pseudospectral collocation (PsC) method}

This method was first proposed by Breda et al [12]. Later, Butcher and Bobrenkov [13] constructed an identical method approaching the problem from the framework of continuous-time approximation [26] and therefore called the technique Chebyshev spectral continuous-time approximation.

Similarly to the PsT method, the PsC method also approximates the OpDE form (9) using the method of weighted residuals and enforces the boundary condition using tau approximation. For the PsC method, base functions $\phi_{j}$ are Lagrange base polynomials defined by the Lobatto-type Chebyshev nodes, which are given as

$$
\zeta_{j}=\cos \left(\frac{(j-1) \pi}{n-1}\right), \quad j=1, \ldots, n .
$$

The main difference between the PsC and PsT methods is in the selection of the test function set. The PsT method uses Legendre polynomials as test functions, while the PsC method applies Dirac-delta functions in the form

$$
\psi_{i}(\zeta)=\delta\left(\zeta-\zeta_{i}\right), \quad i=1, \ldots, n-1 ;
$$

where $\delta$ denotes the Dirac-delta function and $\zeta_{i}$ are the nodes of interpolation (the Lobatto-type Chebyshev nodes). Note that when using Dirac-delta functions (51) as test functions, (16) is equivalent to the system of equations obtained by setting the residual function (15) to zero at the nodes of interpolation. 
The PsC method uses tau approximation and replaces the equation in (16) corresponding to $\zeta=1$ (that is the equation corresponding to $i=n$ ) with the discretized boundary condition (20). Consequently, the final form of the approximate system will be (30)-(33), again. Due to the Dirac-delta test functions, no integration is necessary which simplifies terms in (34)-(35) by one matrix-matrix multiplication. Furthermore, since the Dirac-delta functions are defined on the nodes of interpolation, terms in (34)-(35) are simplified by one more matrix-matrix multiplication. Finally, the finite dimensional approximation $\mathbf{G}(t)$ of operator $\mathcal{G}(t)$ is constructed from the sub-matrices

$$
\mathbf{G}_{i, j}(t)= \begin{cases}\frac{2}{\tau} \mathbf{I} D_{i, j} & i=1, \ldots, n-1 \\ \mathcal{L}(t) \phi_{j} & i=n\end{cases}
$$

Note that for the calculation of $\mathbf{G}(t)$ no inversion and matrix multiplication are necessary, in contrast with the PsT method.

\subsubsection{Spectral Legendre tau (SLT) method}

The SLT method was proposed by Vyasarayani et al. [14] for first-order autonomous RFDEs with constant delays. Similarly to the PsT and PSC methods, the SLT method also approximates the OpDE form (9) using the method of weighted residuals and enforces the boundary condition using tau approximation. The tau approximation is carried out in the same way as for the PsT method, therefore the test functions are $\psi_{i}=P_{i-1}, i=1, \ldots, n-1$. The, main difference between the PsT and SLT methods is in the base functions. While the PsT method uses Lagrange base polynomials defined by the Chebyshev nodes, the SLT method employs Legendre polynomials as base functions (that is $\left.\phi_{j}=P_{j-1}, j=1, \ldots, n\right)$. Consequently, the integral terms in (31)-(32) can be calculated as

$$
\begin{aligned}
\left\langle\phi_{j}, \psi_{i}\right\rangle & =\left\langle P_{j-1}, P_{i-1}\right\rangle, \quad i=1, \ldots, n-1 ; \\
\left\langle\phi_{j}^{\prime}, \psi_{i}\right\rangle & =\left\langle P_{j-1}^{\prime}, P_{i-1}\right\rangle, \quad i=1, \ldots, n-1 .
\end{aligned}
$$

Utilizing the identities and the orthogonality of Legendre polynomials (for details see Appendix A), the integral terms in equations (53)-(54) can be computed in closed form as

$$
\begin{aligned}
\left\langle P_{j-1}, P_{i-1}\right\rangle & =\frac{2}{2 j-1} \delta_{i, j}, \\
\left\langle P_{j-1}^{\prime}, P_{i-1}\right\rangle & = \begin{cases}0 & i>j, \\
2 & (j-i) \bmod 2 \neq 0, \\
0 & (j-i) \bmod 2=0,\end{cases}
\end{aligned}
$$

where $\delta_{i, j}$ is the Kronecker delta. The final form of the approximate system is given again by (30)-(33), using the above formulas.

\subsubsection{Spectral element (SE) method}

The SE method was first proposed in [15], while its further extensions can be found in [27, 28, 29]. In this section, the method is applied and detailed only for the oscillator with distributed delay. However, based on this example, the application of the method for the remaining two RFDEs is straightforward. As a first step, the SE method discretizes the integral term in (47) which, for function segment $\mathbf{z}=\{\mathbf{z}(t): t \in[-\tau, h]\}$ gives the operator equation

$$
\tilde{\mathcal{A}}_{\mathbf{z}}=\mathbf{0}
$$

where the tilde indicates that in general, operator $\tilde{\mathcal{A}}$ is an approximation of operator $\mathcal{A}$. Operator $\tilde{\mathcal{A}}$ is defined as

$$
\tilde{\mathcal{A}} \mathbf{z}=\left\{\dot{\mathbf{z}}(t)-\mathbf{B} \mathbf{z}(t)-\mathbf{C} \sum_{p=1}^{m} \gamma\left(\theta_{p}^{*}\right) \mathbf{z}\left(t-\tau_{p}\right) \frac{w_{p} \tau}{2}: t \in[0, h]\right\}
$$


with

$$
\mathbf{z}(t)=\left[\begin{array}{c}
x(t) \\
\dot{x}(t)
\end{array}\right], \mathbf{B}=\left[\begin{array}{cc}
0 & 1 \\
-a & 0
\end{array}\right], \mathbf{C}=\left[\begin{array}{ll}
0 & 0 \\
b & 0
\end{array}\right]
$$

and $\theta_{p}^{*}=-\tau_{p}=\left(\zeta_{p}^{*}-1\right) \tau / 2$, while $\left\{\zeta_{p}^{*}\right\}_{p=1}^{m}$ and $\left\{w_{p}\right\}_{p=1}^{m}$ are the set of Legendre-Gauss-Lobatto quadrature nodes and weights, respectively. The element length is chosen as $h=\tau / E$, where $E$ is the number of elements.

Note that the PsT, PsC and SLT methods discretize the infinitesimal generator (or operator $\mathcal{G}(t)$ in the non-autonomous case) using $\mathrm{OpDE}(9)$, in order to obtain a system of ordinary differential equations. In contrast, the SE method discretizes the monodromy operator $\mathcal{U}(T)$ using $\mathrm{OpE}(7)$, in order to obtain a discrete mapping. However, all four approximation techniques apply the method of weighted residuals and tau approximation.

The SE method splits the history segment $\mathbf{x}_{0}$ into $E$ number of elements, while the length of $\mathbf{x}^{+}$is equal to the element length $h$. Function segment $\mathbf{z}$ is therefore split into $E+1$ number of elements as

$$
\mathbf{z}^{k}(t)=\left\{\begin{array}{ll}
\mathbf{x}_{0}(t) & \text { if } k \leq 0 \\
\mathbf{x}^{+}(t) & \text { if } k=1
\end{array}, \quad t \in[(k-1) h, k h], \quad k=-E+1,-E+2, \ldots, 1\right.
$$

The elements $\mathbf{z}^{k}$ are then approximated by their Lagrange interpolants as

$$
\tilde{\mathbf{z}}^{k}(t)=\sum_{j=1}^{n} \phi_{j}(t) \mathbf{z}^{k}\left(t_{j}^{k}\right), \quad t \in[(k-1) h, k h],
$$

where $t_{j}^{k} \in[(k-1) h, k h]$. Note that similarly to the PsT and PsC methods the base functions are Lagrange base polynomials again, that is, the SE method is a pseudospectral method. After the substitution of approximate solution segments $\tilde{\mathbf{z}}^{k}$ to (5), one obtains the residual function

$$
\mathbf{r}(t)=\dot{\tilde{\mathbf{z}}}^{1}(t)-\mathbf{B} \tilde{\mathbf{z}}^{1}(t)-\mathbf{C} \sum_{p=1}^{m} \gamma\left(\theta_{p}^{*}\right) \tilde{\mathbf{z}}^{*, p}(t) \frac{w_{p} \tau}{2}, \quad t \in[0, h],
$$

with

$$
\tilde{\mathbf{z}}^{*, p}(t)= \begin{cases}\tilde{\mathbf{z}}^{-r_{p}}\left(t-\tau_{p}\right) & \text { if } t \in\left[0, \alpha_{p}\right], \\ \tilde{\mathbf{z}}^{-r_{p}+1}\left(t-\tau_{p}\right) & \text { if } t \in\left[\alpha_{p}, h\right],\end{cases}
$$

where $\alpha_{p}=\tau_{p} \bmod h$ and $r_{p}=\left[\tau_{p} / h\right]$ is the integer part of $\tau_{p} / h$. Residual function segment $\mathbf{r}=$ $\{\mathbf{r}(t): t \in[0, h]\}$, can be defined by operators as

$$
\mathbf{r}=\mathcal{S} \tilde{\mathbf{z}}^{1}-\sum_{p=1}^{m} \mathcal{Q}_{p} \tilde{\mathbf{z}}^{-r_{p}}-\sum_{p=1}^{m} \mathcal{R}_{p} \tilde{\mathbf{z}}^{-r_{p}+1}
$$

where

$$
\begin{aligned}
\mathcal{S} \tilde{\mathbf{z}}^{1} & = \begin{cases}\dot{\tilde{\mathbf{z}}}^{1}(t)-\mathbf{B} \tilde{\mathbf{z}}^{1}(t) & \text { if } t \in[0, h], \\
\mathbf{0} & \text { otherwise, }\end{cases} \\
\mathcal{Q}_{p} \tilde{\mathbf{z}}^{-r_{p}} & = \begin{cases}\mathbf{C} \gamma\left(\theta_{p}^{*}\right) \tilde{\mathbf{z}}^{-r_{p}}\left(t-\tau_{p}\right) \frac{w_{p} \tau}{2} & \text { if } t \in\left[0, \alpha_{p}\right], \\
\mathbf{0} & \text { otherwise },\end{cases} \\
\mathcal{R}_{p} \tilde{\mathbf{z}}^{-r_{p}+1} & = \begin{cases}\mathbf{C} \gamma\left(\theta_{p}^{*}\right) \tilde{\mathbf{z}}^{-r_{p}+1}\left(t-\tau_{p}\right) \frac{w_{p} \tau}{2} & \text { if } t \in\left[\alpha_{p}, h\right], \\
\mathbf{0} & \text { otherwise }\end{cases}
\end{aligned}
$$

The element-wise, linear coordinate transformation

$$
\zeta^{k}=\frac{2(t-(k-1) h)}{h}-1, \quad k=-E+1,-E+2, \ldots, 1
$$


defines a shift from the global coordinate $t \in[-\tau, h]$ to local coordinates $\zeta^{k} \in[-1,1], k=-E+$ $1,-E+2, \ldots, 1$. Since the domains of all the local coordinates $\zeta^{k}$ are the same, the upper indices are dropped in the sequel. Coordinate transformation (68) changes operators in (65)-(67) as

$$
\begin{aligned}
\mathcal{S} \tilde{\mathbf{z}}^{1} & = \begin{cases}\frac{2}{h} \tilde{\mathbf{z}}^{\prime 1}(\zeta)-\mathbf{B} \tilde{\mathbf{z}}^{1}(\zeta) & \text { if } \zeta \in[-1,1] \\
\mathbf{0} & \text { otherwise },\end{cases} \\
\mathcal{Q}_{p} \tilde{\mathbf{z}}^{-r_{p}} & = \begin{cases}\mathbf{C} \gamma\left(\theta_{p}^{*}\right) \tilde{\mathbf{z}}^{-r_{p}}\left(\zeta+2-\beta_{p}\right) \frac{w_{p} \tau}{2} & \text { if } \zeta \in\left[-1,-1+\beta_{p}\right], \\
\mathbf{0} & \text { otherwise }\end{cases} \\
\mathcal{R}_{p} \tilde{\mathbf{z}}^{-r_{p}+1} & = \begin{cases}\mathbf{C} \gamma\left(\theta_{p}^{*}\right) \tilde{\mathbf{z}}^{-r_{p}+1}\left(\zeta-\beta_{p}\right) \frac{w_{p} \tau}{2} & \text { if } \zeta \in\left[-1+\beta_{p}, 1\right], \\
\mathbf{0} & \text { otherwise }\end{cases}
\end{aligned}
$$

where $\beta_{p}=2 \alpha_{p} / h$. The application of the method of weighted residuals gives

$$
\left\langle\mathcal{S} \tilde{\mathbf{z}}^{1}, \psi_{i}\right\rangle-\sum_{p=1}^{m}\left\langle\mathcal{Q}_{p} \tilde{\mathbf{z}}^{-r_{p}}, \psi_{i}\right\rangle-\sum_{p=1}^{m}\left\langle\mathcal{R}_{p} \tilde{\mathbf{z}}^{-r_{p}+1}, \psi_{i}\right\rangle=\mathbf{0}, \quad i=1, \ldots, n
$$

which can be expanded as

$$
\sum_{j=1}^{n} \mathbf{S}_{i, j} \tilde{\mathbf{z}}^{1, j}-\sum_{p=1}^{m} \sum_{j=1}^{n} \mathbf{Q}_{i, j}^{p} \tilde{\mathbf{z}}^{-r_{p}, j}-\sum_{p=1}^{m} \sum_{j=1}^{n} \mathbf{R}_{i, j}^{p} \tilde{\mathbf{z}}^{-r_{p}+1, j}=\mathbf{0}, \quad i=1, \ldots, n ;
$$

with

$$
\begin{aligned}
& \mathbf{S}_{i, j}=\int_{-1}^{1}\left(\frac{2}{h} \mathbf{I} \phi_{j}^{\prime}(\zeta)-\mathbf{B} \phi_{j}(\zeta)\right) \psi_{i}(\zeta) \mathrm{d} \zeta=\frac{2}{h} \mathbf{I} \sum_{q=1}^{n} F_{i, q} D_{q, j}-\mathbf{B} F_{i, j}, \\
& \mathbf{Q}_{i, j}^{p}=\mathbf{C} \gamma\left(\theta_{p}^{*}\right) \frac{w_{p} \tau}{2} \int_{-1}^{-1+\beta_{p}} \phi_{j}\left(\zeta+2-\beta_{p}\right) \psi_{i}(\zeta) \mathrm{d} \zeta=\mathbf{C} \gamma\left(\theta_{p}^{*}\right) \frac{w_{p} \tau}{2} \sum_{q=1}^{n} F_{i, q}^{Q, p} L_{q, j}^{Q, p}, \\
& \mathbf{R}_{i, j}^{p}=\mathbf{C} \gamma\left(\theta_{p}^{*}\right) \frac{w_{p} \tau}{2} \int_{-1+\beta_{p}}^{1} \phi_{j}\left(\zeta-\beta_{p}\right) \psi_{i}(\zeta) \mathrm{d} \zeta=\mathbf{C} \gamma\left(\theta_{p}^{*}\right) \frac{w_{p} \tau}{2} \sum_{q=1}^{n} F_{i, q}^{R, p} L_{q, j}^{R, p},
\end{aligned}
$$

where $F_{i, q}$ and $D_{q, j}$ are defined under (35) and (27), respectively, while

$$
\begin{aligned}
& F_{i, q}^{Q, p}=\psi_{i}\left(\frac{\beta_{p}}{2}\left(\zeta_{q}^{*}+1\right)-1\right) \frac{w_{q} \beta_{p}}{2}, \quad L_{q, j}^{Q, p}=\phi_{j}\left(\frac{\beta_{p}}{2}\left(\zeta_{q}^{*}-1\right)+1\right), \\
& F_{i, q}^{R, p}=\psi_{i}\left(\frac{\left(2-\beta_{p}\right) \zeta_{q}^{*}+\beta_{p}}{2}\right) \frac{w_{q}\left(2-\beta_{p}\right)}{2}, \quad L_{q, j}^{R, p}=\phi_{j}\left(\frac{\left(2-\beta_{p}\right) \zeta_{q}^{*}-\beta_{p}}{2}\right) .
\end{aligned}
$$

Note, that according to (4), the boundary condition $\mathbf{x}_{0}(0)=\mathbf{x}^{+}(0)$ has to be enforced, that is equation

$$
\tilde{\mathbf{z}}^{0}(1)=\tilde{\mathbf{z}}^{1}(-1)
$$

has to be satisfied. By assuming Legendre-Gauss-Lobatto node set for interpolation and introducing notation $\tilde{\mathbf{z}}^{k, j}=\tilde{\mathbf{z}}^{k}\left(\zeta_{j}^{*}\right)$, (79) gives

$$
\tilde{\mathbf{z}}^{0, n}=\tilde{\mathbf{z}}^{1,1} .
$$

Consequently, if the history segment $\mathbf{x}_{0}$ is known, one ends up with $n$ number of unknowns $\left(\tilde{\mathbf{z}}^{1,1}, \tilde{\mathbf{z}}^{1,2}, \ldots, \tilde{\mathbf{z}}^{1, n}\right)$ and $n+1$ number of equations given by (73) and (80). The SE method applies the tau approximation in the same way as in Section 4.2, hence in (73) the equation with index $i=n$ is replaced by (80) and the test functions are set to be the Legendre polynomials $\left(\psi_{i}=P_{i-1}\right)$. Using (73) with $i=1, \ldots, n-1$ and $(80), \tilde{\mathbf{z}}^{1, j}, j=1, \ldots, n$ can be calculated for any initial function segment $\mathbf{x}_{0}$. Due to the Legendre-Gauss-Lobatto node set

$$
\tilde{\mathbf{z}}^{k, n}=\tilde{\mathbf{z}}^{k+1,1}, \quad k=-E+1,-E+2, \ldots,-1 ;
$$




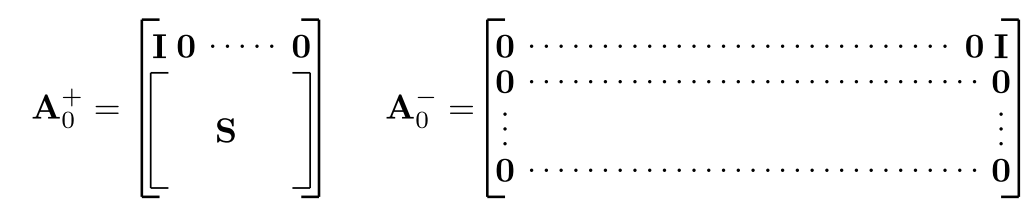

$$
\begin{aligned}
& \text { if } r_{p}=0 \text { and } p>0 \text { : }
\end{aligned}
$$

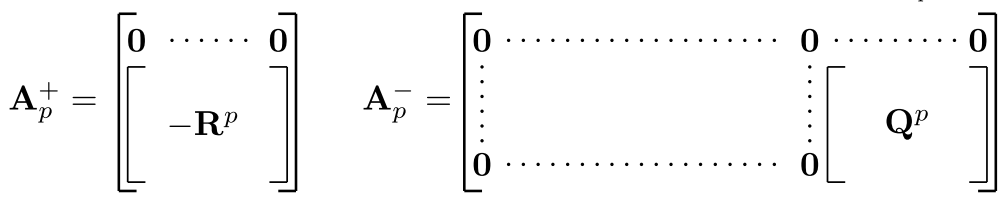

$$
\begin{aligned}
& \text { if } r_{p}>0 \text { and } p>0 \text { : } \\
& \mathbf{A}_{p}^{+}=\mathbf{0}
\end{aligned}
$$

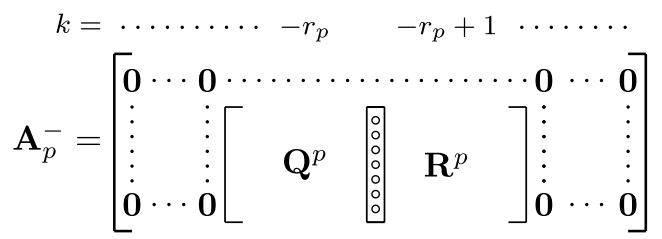

Figure 3: The structure of matrices $\mathbf{A}_{p}^{+}$and $\mathbf{A}_{p}^{-}$

hold. Taking this into consideration, equations (73) $i=1, \ldots, n-1$ and (80) define the mapping

$$
\mathbf{A}^{+} \mathbf{X}^{+}=-\mathbf{A}^{-} \mathbf{X}^{0}
$$

where

$$
\mathbf{A}^{+} \in \mathbb{R}^{2 n \times 2 n}, \mathbf{A}^{-} \in \mathbb{R}^{2 n \times(2 E(n-1)+2)}, \mathbf{X}^{+}=\left[\mathbf{z}^{1, j}\right]_{j=1}^{n}, \mathbf{X}^{0}=\left[\mathbf{X}_{l}^{0}\right]_{l=1}^{E(n-1)+1},
$$

with

$$
\mathbf{X}_{l}^{0}=\tilde{\mathbf{z}}^{k, j}, \quad k=\left\{\begin{array}{ll}
1-E & \text { if } l=1 \\
{\left[\frac{l-2}{n-1}\right]+1-E} & \text { otherwise }
\end{array}, \quad j=\left\{\begin{array}{ll}
1 & \text { if } l=1 \\
l-(k-1+E)(n-1) & \text { otherwise }
\end{array} .\right.\right.
$$

Matrices $\mathbf{A}^{-}$and $\mathbf{A}^{+}$can be calculated as

$$
\mathbf{A}^{-}=-\sum_{p=0}^{m} \mathbf{A}_{p}^{-}, \quad \mathbf{A}^{+}=\sum_{p=0}^{m} \mathbf{A}_{p}^{+}
$$

where the structure of $\mathbf{A}_{p}^{+}$and $\mathbf{A}_{p}^{-}$are shown in Figure 3. The elements of submatrices $\mathbf{S}, \mathbf{Q}^{p}$, and $\mathbf{R}^{p}$ are defined according to (74)-(76). For the case $r_{p}>0, p>0$, the last two columns of $\mathbf{Q}^{p}$ and the first two columns of $\mathbf{R}^{p}$ within matrix $\mathbf{A}^{-}$overlap. The overlapped parts are highlighted by circles in Figure 3, and the overlapped elements are merged by summation.

Note that $\mathbf{X}^{0}$ and $\mathbf{X}^{h}=\left[\mathbf{X}_{l}^{h}\right]_{l=1}^{E(n-1)+1}$ define piecewise Lagrange interpolants of $\mathbf{x}_{0}$ and $\mathbf{x}_{h}$, respectively, where the elements of $\mathbf{X}^{h}$ are given by

$$
\mathbf{X}_{l}^{h}= \begin{cases}\mathbf{X}_{l+n-1}^{0} & \text { if } 0<l \leq(E-1)(n-1) \\ \mathbf{z}^{1, l-(E-1)(n-1)} & \text { if }(E-1)(n-1)<l \leq E(n-1)+1 .\end{cases}
$$

Since (47) is autonomous, the principal period can be set to $T=h$. The mapping between $\mathbf{X}^{h}$ and $\mathbf{X}^{0}$ thus define a matrix approximation $\mathbf{U}(T)$ of monodromy operator $\mathcal{U}(T)$. The structure of $\mathbf{U}(T)$ is shown in Figure 4, where $\boldsymbol{\Theta}$ represents a null matrix. The approximate system is stable if and only if all eigenvalues (multipliers) $\mu$ of $\mathbf{U}(T)$ have modulus less than one. Using the relation $\mu=\mathrm{e}^{\lambda T}$ the real parts of the characteristic exponents can be calculated as

$$
\operatorname{Re}(\lambda)=\frac{1}{\tau} \ln \sqrt{\operatorname{Re}^{2}(\mu)+\operatorname{Im}^{2}(\mu)},
$$




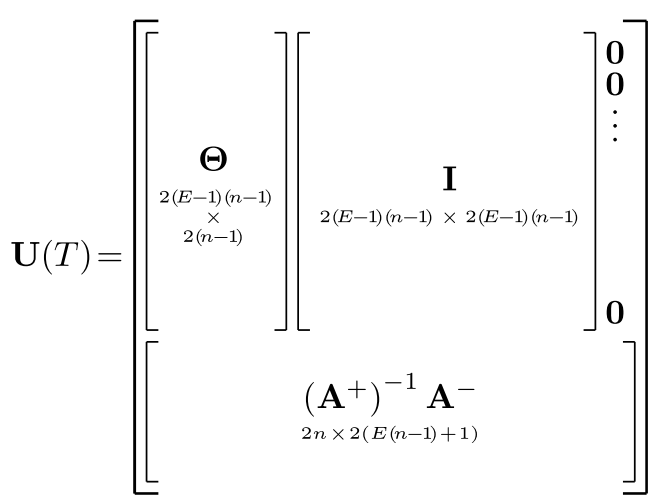

Figure 4: Structure of the matrix approximation $\mathbf{U}(T)$ of monodromy operator $\mathcal{U}(T)$ depicted with the corresponding matrix dimensions

however the corresponding imaginary parts cannot be uniquely determined from the characteristic multipliers. Note that the inverse of $\mathbf{A}^{+}$has to be recomputed each time the system parameters are updated. For the results, presented in the next subsection $m=n$ is used ( $m$ is the number of quadrature points used for the approximation of the distributed delay in (58)). These results are obtained for $E=1$, that is the effect of element-wise refinement is not investigated. Note however, that element-wise refinement can be applied also for the PsT, PsC and SLT methods. An example for such element-wise refinement is given in [30] for the Chebyshev spectral continuous-time approximation.

\subsection{Results}

Results for the comparison of the PsT, PsC, SLT and SE methods are given in Tables 1-5. The approximate boundaries of stability were determined as follows. Eigenvalues of matrix $\mathbf{G}$ (or $\mathbf{U}(T)$ ) were computed for a series of system parameters on an equidistant grid of a particular domain in the plane of system parameters. Eigenvalues, having the largest real part (or largest absolute value) were stored for each gridpoint. A 3-dimensional surface was fitted on these eigenvalues over the parameter plane using the "contour" function of Matlab. The approximate borders of stability were given by the zero-level curve (or level curve at 1) of this 3-dimensional surface. Note that efficiency of this algorithm can be improved if only the critical eigenvalues are calculated (e.g. see [31]). Efficiency could also be increased if non-uniform grid is used in the parameter plane (for such methods see [32] and [33]). The convergence of the rightmost characteristic exponents were investigated for parameter combinations given in Table 1.

Table 2 presents the stability boundaries for the investigated RFDEs using different methods with an increasing order $n$ of approximation. It is observed, that the convergence of stability boundaries is almost exactly the same for the PsT, the SE and the SLT methods, while the PsC method has slower convergence for stability.

Table 3 shows the real part of the error of the rightmost characteristic exponent as a function of the order of approximation. Results show that the SE method tends to have the highest rate while the PsC method tends to have the lowest rate of convergence. It is interesting to note that the points corresponding to the PsT method and the SLT method coincide for smaller $n$ values. Their convergence rates are close to that of the SE method in case of the Hayes equation and the oscillator with distributed delay, while in case of the oscillator with two delays their convergence rates are close to that of the PsC method. Tables 2-3 can be used for the comparison of methods based on their convergence with respect to $n$. However, in practice the time necessary for the computation of the stability charts is also important. Clearly, the time demand of a method depends on its realization as a particular algorithm. A reliable base for comparison could be the number of necessary floating point operations, however, such analysis is not performed in this paper. Here the computational demand of the methods is characterised by the time necessary for the computation of stability charts.

Table 4 shows the computational time of stability charts using $200 \times 200$ gridpoints on the plane of system parameters. The computational time is presented as a function of approximation order 


\begin{tabular}{|c|c|c|c|c|c|c|}
\hline & \multicolumn{2}{|c|}{ Hayes equation } & \multicolumn{2}{c|}{ Osc. w. two delays } & \multicolumn{2}{c|}{ Osc. w. distr. delay } \\
\cline { 2 - 7 } & $a$ & $b$ & $\tau_{1} / \pi$ & $\tau_{2} / \pi$ & $a / \pi^{2}$ & $b / \pi^{2}$ \\
\hline \hline A & -10 & 5 & 1.2 & 0.9 & 10 & -5 \\
\hline B & -5 & -10 & 2.4 & 1.1 & 18 & 18 \\
\hline C & 0.5 & -1 & 3 & 1.5 & 15 & 30 \\
\hline
\end{tabular}

Table 1: Investigated points on the plane of system parameters

for different equations and different methods. For the calculation of stability charts, the software MATLAB was used. During the construction of MATLAB codes the authors strived to increase the efficiency evenly for algorithms corresponding to the investigated methods in order to provide a base of a fair comparison. Based on the structure of the methods under comparison some preliminary estimations can be made on their speed. In all four methods, the majority of computational time is spent on the determination of the eigenvalues of $\mathbf{G}$ (for the PsT, PsC and SLT methods) or $\mathbf{U}$ (for the SE method). Note that the herein presented algorithm calculates all the eigenvalues of these matrices, although for stability only the critical eigenvalue is of interest. It is expected that the structure of the matrix has no significant effect on the time of eigenvalue computation. However, the inversion of matrices $\mathbf{A}^{+}$and $\mathbf{N}$ cost considerable time which has to be done repeatedly in case of the SE method and once in case of the PsT and SLT method. Note also that under updated system parameters, the PsC method has to carry out one less matrix-matrix multiplication compared to the other methods. This matrix multiplication however, has small effect on the overall computational time. The results presented in Table 4 match with the above discussion, that is the SE method requires more computational effort, while the rest of the methods have similar computational demand. In practice, a limit is set for the relative error of the real part of the rightmost exponent and the order of approximation is increased until this limit is reached. Therefore a more useful diagram can be constructed by the combination of Table 3 and Table 4, which is shown in Table 5 .

Table 5 shows parametric "curves" with the running parameter $n$ on the plane of computational time and error of the real part of the rightmost characteristic exponent. The farthest to the left a curve is placed on this plane the better time efficiency the corresponding method has. Note that while the computational time corresponds to a stability chart, the error corresponds to a particular point of this chart. It is therefore assumed that the time which is necessary for the calculation of the exponents is the same in all points of the parameter plane. The results of Table 5 show, that for the Hayes equation the PsT method, for the oscillator with two delays the PsC method, while for the oscillator with distributed delay the SLT method is the most time-efficient. The SE method has the least timeefficiency for the investigated cases. Note, that the SE method can approximate only the real parts of the exponents according to (87), therefore, it cannot be used for applications where the location of exponents is of interest on the complex plane (e.g. continuous pole placement [34]). Also note that in case of pseudospectral methods the variables of the discretized system are distinct points of the history function, which can be advantageous in some applications. Furthermore, it should be mentioned that, to the best knowledge of the authors, detailed theoretical convergence analysis does not exist for the PsT and SE methods. However, for the PsC and the SLT methods, precise theoretical convergence analysis is provided in [35] and in [36], respectively.

\section{Application for time-periodic systems}

The proposed method can also be applied to time-periodic systems based on the Floquet theory. As it is detailed in Section 4, the PsT method approximates RFDE (1) by the ODE (33). When the RFDE is time-periodic, stability is determined by the characteristic multipliers of the monodromy operator $\mathcal{U}(T)$. A matrix approximation $\mathbf{U}(T)$ of the monodromy operator can be defined by the mapping

$$
\mathbf{X}(T)=\mathbf{U}(T) \mathbf{X}(0) .
$$




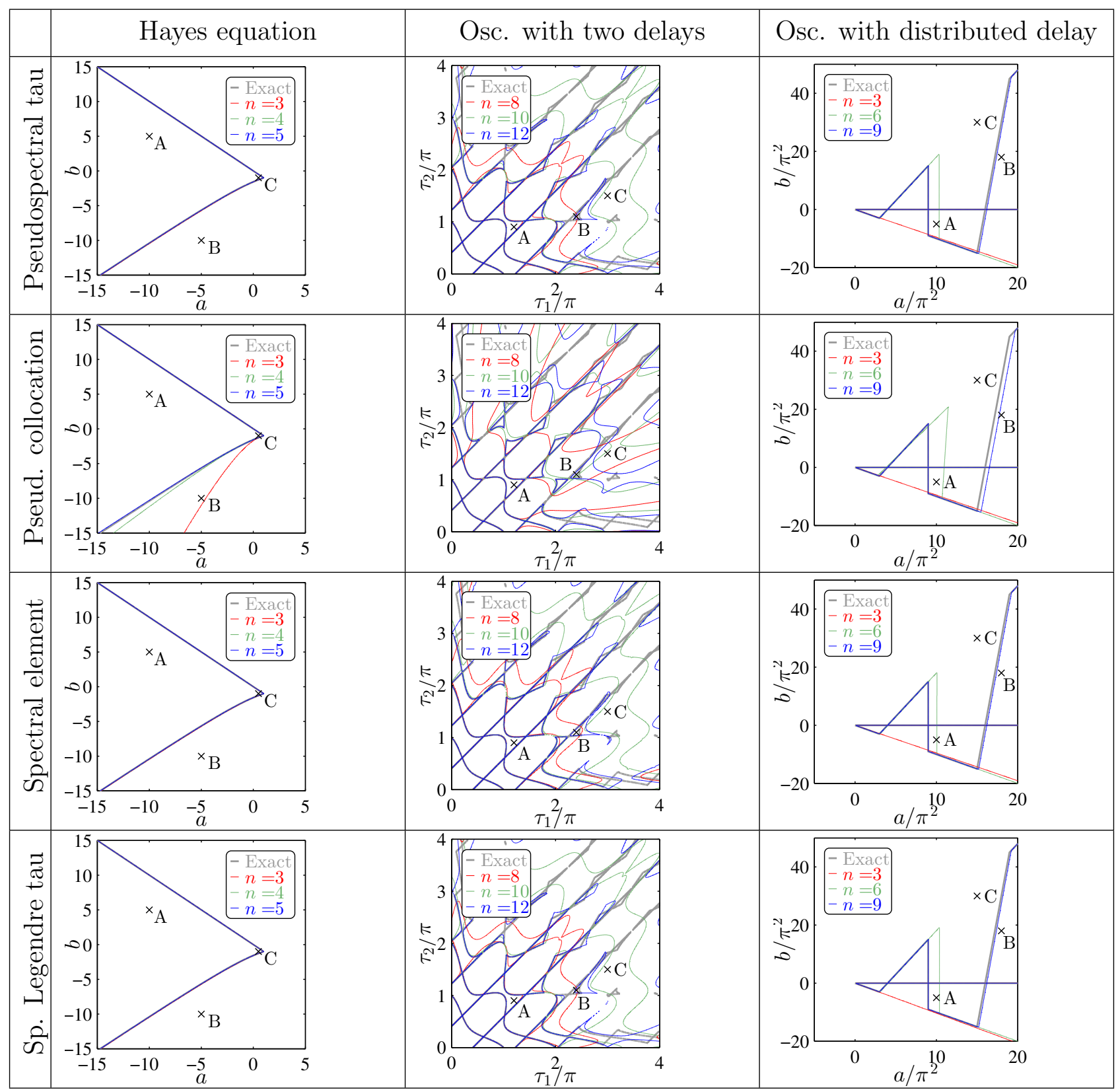

Table 2: Convergence of stability boundaries using different methods for different RFDEs 


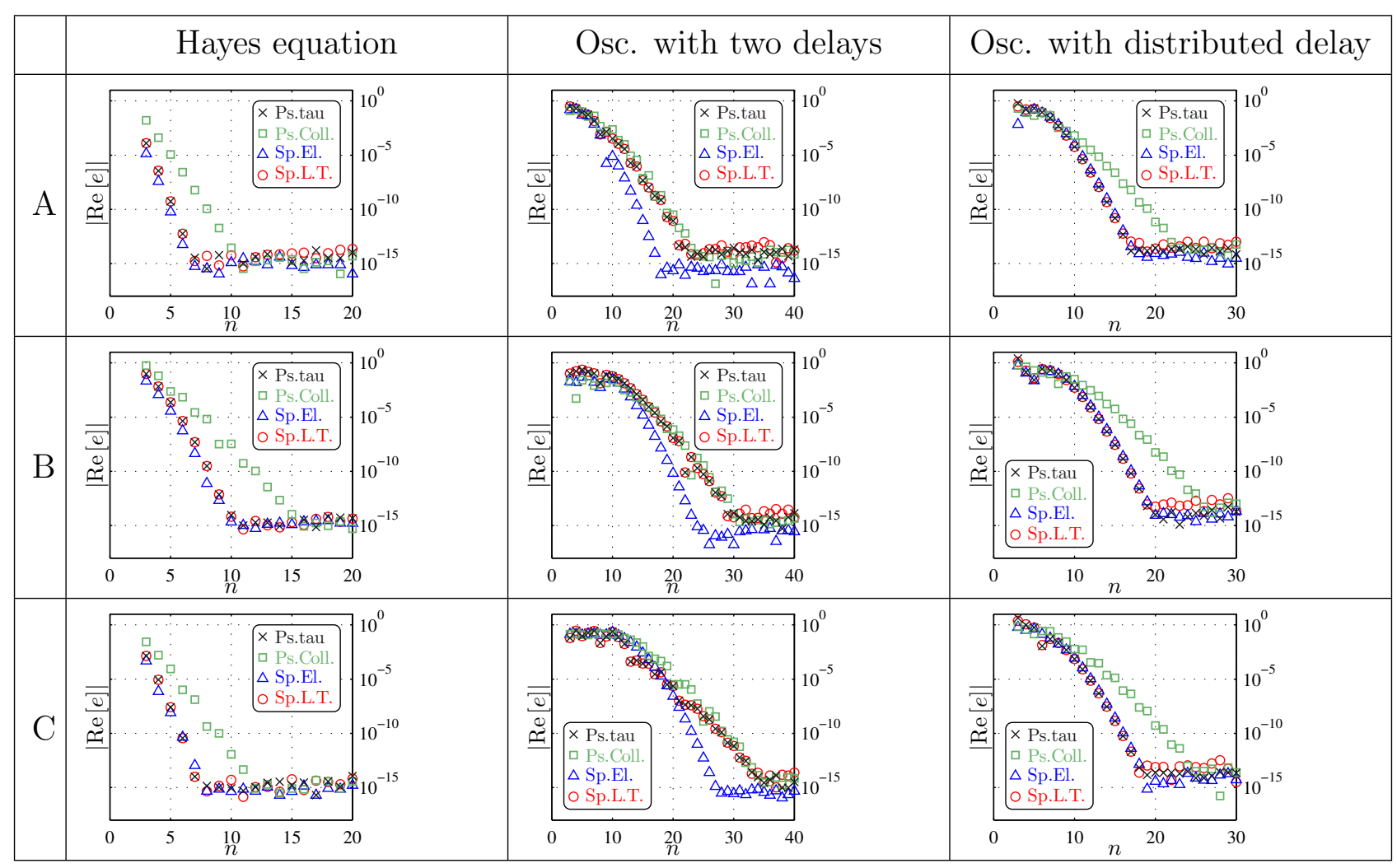

Table 3: The convergence of the real part of the rightmost exponent using different methods for different RFDEs and system parameters according to Table 1

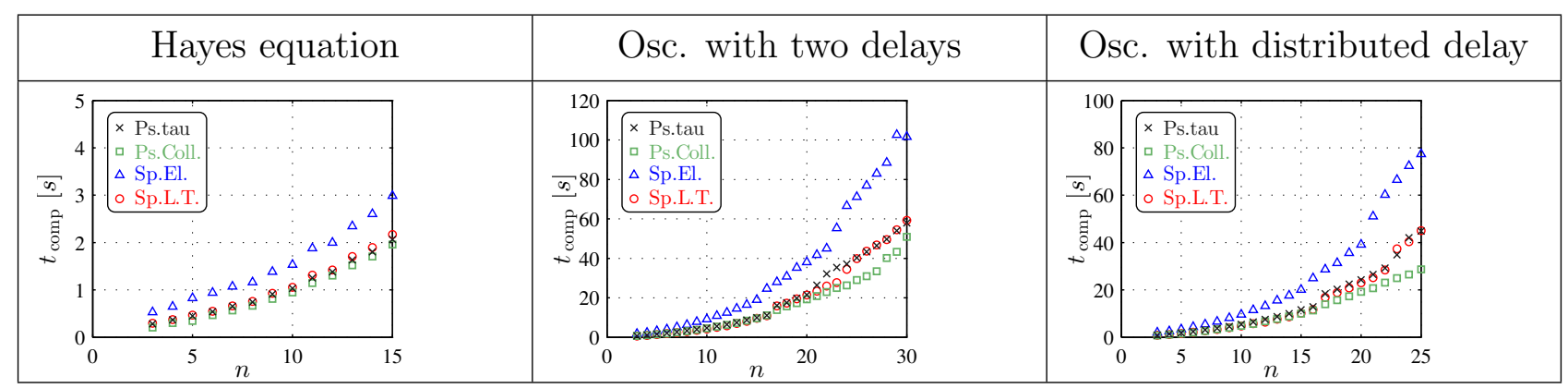

Table 4: Necessary time for the computation of stability charts based on the calculation of approximate characteristic exponents/multipliers in $200 \times 200$ points of the plane of system parameters 


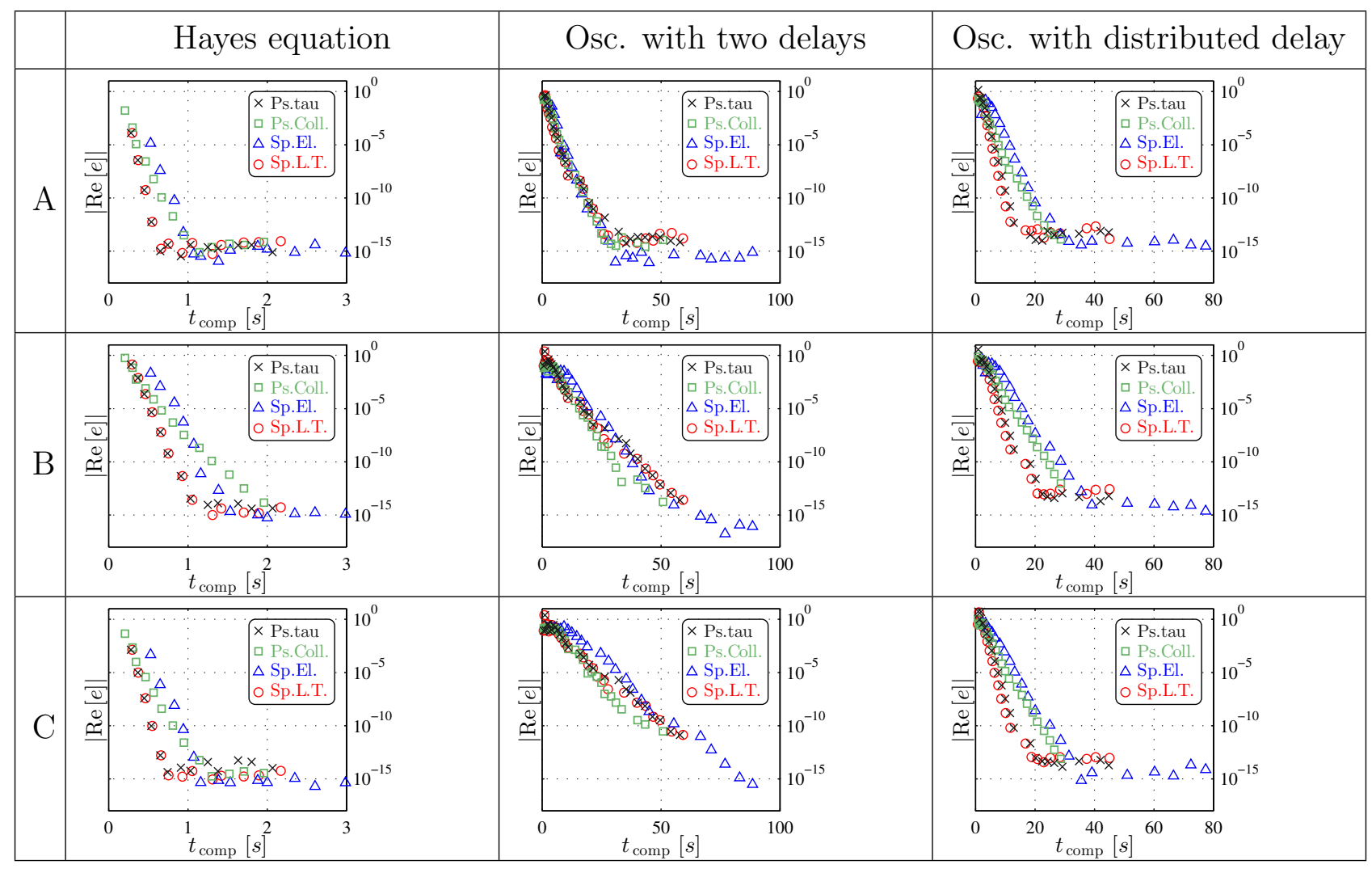

Table 5: Time efficiency of different methods for different RFDEs and system parameters according to Table 1

However, in general, $\mathbf{U}(T)$ cannot be calculated in closed form. A simple approximation for $\mathbf{U}(T)$ can be given via the approximation of the time-periodic ODE (33) by a series of autonomous ODEs in the form

$$
\dot{\mathbf{V}}_{k}(t)=\mathbf{G}_{k} \mathbf{V}_{k}(t) \quad t \in((k-1) \Delta t, k \Delta t], \quad k=1,2, \ldots, N ;
$$

where $\mathbf{V}_{k}=\left\{\mathbf{V}_{k}(t): t \in((k-1) \Delta t, k \Delta t]\right\}$ solution segments are connected by boundary conditions

$$
\mathbf{V}_{k}(k \Delta t)=\mathbf{V}_{k+1}(k \Delta t), \quad k=1,2, \ldots, N-1 .
$$

In (89), the time step is $\Delta t=T / N$ with $N \in \mathbb{N}$ being the period resolution, while

$$
\mathbf{G}_{k}=\frac{1}{\Delta t} \int_{(k-1) \Delta t}^{k \Delta t} \mathbf{G}(\eta) \mathrm{d} \eta
$$

is a piecewise constant approximation of $\mathbf{G}(t)$. The approximation of $\mathbf{U}(T)$ is then given by

$$
\mathbf{U}(T) \approx \tilde{\mathbf{U}}=\mathbf{e}^{\mathbf{G}_{N}^{*}} \mathbf{e}^{\mathbf{G}_{N-1}^{*}} \cdots \mathbf{e}^{\mathbf{G}_{1}^{*}},
$$

where

$$
\mathbf{G}_{k}^{*}=\mathbf{G}_{k} \Delta t=\mathbf{N}^{-1} \int_{0}^{\Delta t} \mathbf{M}(\eta+(k-1) \Delta t) \mathrm{d} \eta, \quad k=1,2, \ldots, N .
$$

Note that the monodromy operator is approximated in two steps. First, (9) is discretized by the PsT method, then (33) is approximated by (89). In contrast, the direct approximation of the solution operator is more sound, which can be carried out e.g. by the SE method or the collocation technique in [37].

\subsection{Examples}

Two examples are shown for the application of this method. Namely, stability charts are determined for the delayed Mathieu equation and for an oscillator with time-periodic delay. 


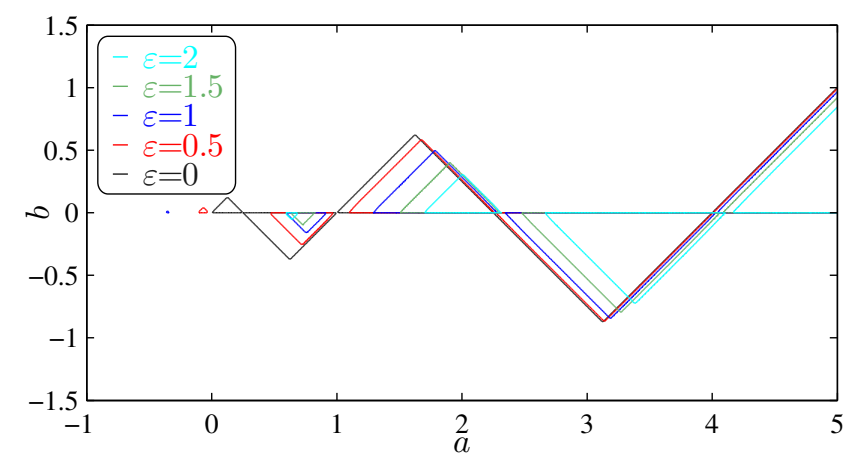

Figure 5: Stability chart of the delayed Mathieu equation for $\tau=T=2 \pi, n=12$ and $N=15$

\subsubsection{Delayed Mathieu equation}

The first-order form of the delayed Mathieu equation is

$$
\dot{\mathbf{x}}(t)=\left(\mathbf{B}+\mathbf{B}_{T}(t)\right) \mathbf{x}(t)+\mathbf{C} \mathbf{x}(t-\tau),
$$

where

$$
\mathbf{B}_{T}(t)=\varepsilon\left(\begin{array}{cc}
0 & 0 \\
-\cos \left(\frac{2 \pi t}{T}\right) & 0
\end{array}\right),
$$

while matrices $\mathbf{B}$ and $\mathbf{C}$ are given in (59). Derivation of the exact stability boundaries for this equation can be found in [38]. Using the PsT method the discretization of (94) is carried out according to (37)(38), where now

$$
\mathcal{L}(t) \phi_{j}=\left(\mathbf{B}+\mathbf{B}_{T}(t)\right) \phi_{j}(1)+\mathbf{C} \phi_{j}(-1) .
$$

Note, that for the computation of $\tilde{\mathbf{U}}$, integral terms in (93) have to be calculated only once, even for varying $a, b, \varepsilon, \tau$ system parameters. By increasing $n$ and $N$, one can observe the convergence of the approximate stability chart to the analytical one. In Figure 5, the converged stability boundaries are shown for fixed $\tau$ and $T$ parameters.

\subsubsection{Oscillator with one time-periodic delay}

The first-order form of this oscillator is given by

$$
\dot{\mathbf{x}}(t)=\mathbf{B} \mathbf{x}(t)+\mathbf{C} \mathbf{x}(t-\rho(t)),
$$

where matrices $\mathbf{B}$ and $\mathbf{C}$ are defined in (59), and $\rho(t+T)=\rho(t)$ with $\rho(t) \geq 0 \forall t$. Again, discretization of (97) is performed according to (37)-(38), where now

$$
\mathcal{L}(t) \phi_{j}=\mathbf{B} \phi_{j}(1)+\mathbf{C} \phi_{j}\left(1-\frac{2 \rho(t)}{\tau}\right)
$$

with $\tau$ being the maximum value of the delay. In Figure 6 approximate stability charts are shown for the time-periodic delay

$$
\rho(t)=2 \pi\left(1+\varepsilon \cos \left(\frac{2 \pi t}{T}\right)\right) .
$$

After the comparison of the results presented in Figure 6 and the results shown in Fig. 4.11 in Chapter 4 of [19], one can observe that the boundaries of stability, determined by the PsT method, converge to those determined by the semi-discretization method. 

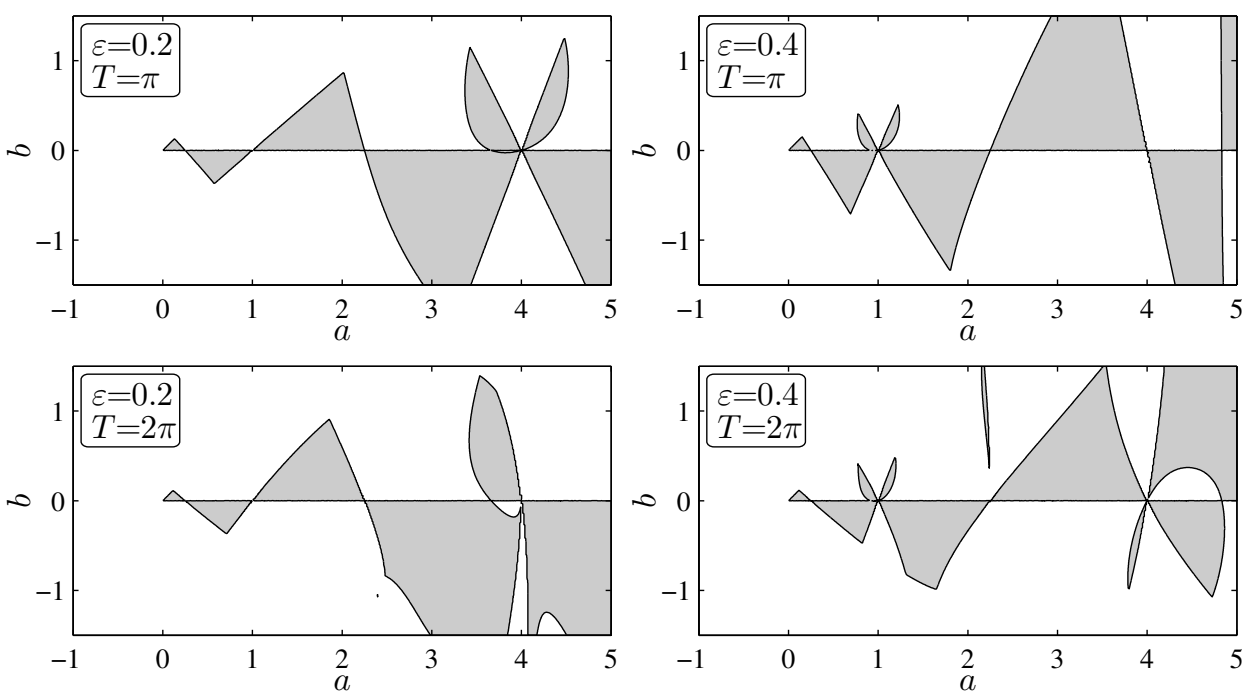

Figure 6: Stability charts of the oscillator with time-periodic delay for $n=15$ and $N=20$

\section{Conclusions}

In this paper, the competitiveness of the PsT method against recently developed highly efficient numerical methods was shown with respect to the stability analysis and ODE approximation of RFDEs. The properties of the compared methods were presented by means of their application to three linear autonomous RFDEs. The results of Section 5 imply that the PsT and SLT methods have similar high time efficiency, while the PsC and SE methods have a bit lower time efficiency for the determination of stability charts in the plane of system parameters. It is also shown, that the PsT and SLT methods approximate the rightmost root of ODEs with similar high convergence rate, while the PsC method has lower rate of convergence. Here the authors note that the convergence rate of the PsT and SLT methods is higher than that of the PsC method not only for the rightmost root but also for the finite dimensional approximation of the complete spectrum of the RFDE. This property is not detailed here but is experienced by the authors through several numerical experiments. Based on the results of Section 5, the authors believe, that the PsT method is a useful, highly efficient tool for the stability analysis and ODE approximation of linear autonomous RFDEs. Furthermore, the results of Section 6 imply that the PsT method can also be used for the ODE approximation of time-periodic RFDEs.

\section{Acknowledgments}

This research project was supported by the Hungarian National Science Foundation under grant OTKA-K105433. The research leading to these results has received funding from the European Research Council under the European Union's Seventh Framework Programme (FP7/2007-2013) / ERC Advanced grant agreement $\mathrm{N}^{\circ} 340889$. The authors thank prof. Janos Turi for his helpful suggestions and comments. 


\section{Appendices}

\section{Appendix A Legendre polynomials}

Definition of Legendre polynomials by Bonnet's recursion formula:

$$
\begin{aligned}
& P_{0}(\zeta)=1 \\
& P_{1}(\zeta)=\zeta \\
& P_{j}(\zeta)=\frac{2 j-1}{j} \zeta P_{j-1}(\zeta)-\frac{j-1}{j} P_{j-2}(\zeta) \quad j=2,3, \ldots
\end{aligned}
$$

Some properties of Legendre polynomials:

- orthogonality: $\int_{-1}^{1} P_{j}(\zeta) P_{i}(\zeta) \mathrm{d} \zeta=\frac{2}{2 j+1} \delta_{i, j}$

- $P_{j}(1)=1$ and $P_{j}(-1)=(-1)^{j}$

Recursion formula for the first derivative of Legendre polynomials:

$$
P_{j}^{\prime}(\zeta)=(2 j-1) P_{j-1}(\zeta)+P_{j-2}^{\prime}(\zeta) \quad j=2,3, \ldots
$$

Due to the above formula and the orthogonality property of Legendre polynomials:

$$
\int_{-1}^{1} P_{j}^{\prime}(\zeta) P_{i}(\zeta) \mathrm{d} \zeta= \begin{cases}0 & \text { if } i>j \\ 2 & \text { if }(j-i) \bmod 2 \neq 0 \\ 0 & \text { if }(j-i) \bmod 2=0\end{cases}
$$

\section{Appendix B Legendre-Gauss-Lobatto quadrature}

The Legendre-Gauss-Lobatto quadrature approximates a definite integral by a sum as

$$
I=\int_{a}^{b} x(t) \mathrm{d} t \approx \tilde{I}=\sum_{q=1}^{n} x\left(t_{q}\right) w_{q},
$$

where $t_{q}=\frac{a-b}{2} \zeta_{q}^{*}+\frac{a+b}{2}$ with $\zeta_{q}^{*}$ and $w_{q}$ being the quadrature nodes and weights, respectively. The Legendre-Gauss-Lobatto quadrature gives exact results for all polynomials with maximum order $2 n-3$. The quadrature nodes are the roots of $\left(1-\zeta^{2}\right) P_{n-1}^{\prime}(\zeta)$, that is $-1,1$ and the roots of the first derivative of the Legendre polynomial of order $(n-1)$. The quadrature weights are given by

$$
w_{q}= \begin{cases}\frac{2}{n(n-1)} & \text { if } q=1, n \\ \frac{2}{n(n-1) P_{n-1}^{2}\left(\zeta_{q}^{*}\right)} & \text { if } q=2,3, \ldots, n-1 .\end{cases}
$$

\section{Appendix C Chebyshev points and polynomials}

\section{Theorem}

Consider the Lagrange interpolant $\tilde{x}(\theta)$ of $x(\theta)$ on the domain $\theta \in[a, b]$, using the node set $\left\{\theta_{j}\right\}_{j=1}^{n} \subset$ $[a, b]$. Assume, that $x(t)$ is $C^{n}$ on the given interval. Then at any $\theta$ point of $[a, b]$, the error $E_{n}(\theta)=$ $x(\theta)-\tilde{x}(\theta)$ of interpolation is given by the formula

$$
E_{n}(\theta)=-\frac{x^{(n)}\left(\theta^{*}\right)}{n !} \omega_{n}(\theta), \quad \omega_{n}(\theta)=\prod_{j=1}^{n}\left(\theta-\theta_{j}\right),
$$

where $\theta^{*} \in[a, b]$ is a constant which depends on the value of $\theta$. 


\section{Theorem}

Let $[a, b]$ a fixed interval. For a $p_{n}(\theta)$ n-order polynomial with leading coefficient $1,\left|p_{n}(\theta)\right|_{C[a, b]} \leq$ $\left|q_{n}(\theta)\right|_{C[a, b]}$ for all $q_{n}(\theta) n$-order polynomial with leading coefficient 1 if and only if $p_{n}(\theta)$ has at least $n+1$ distinct absolute extrema on $[a, b]$. On these points the absolute value of $p_{n}(\theta)$ is the same, while the sign of its value alternates.

\section{Theorem}

There is only one $p_{n}(\theta) n$-order polynomial with leading coefficient 1 on $[a, b]$, for which $\left|p_{n}(\theta)\right|_{C[a, b]} \leq$ $\left|q_{n}(\theta)\right|_{C[a, b]}$ for all $q_{n}(\theta)$-order polynomial with leading coefficient 1 .

\section{Theorem}

On the domain $[-1,1]$ the function

$$
\tilde{T}_{n+1}(\theta)=\frac{\cos (n \arccos (\theta))}{2^{n-1}}, \quad n \geq 1
$$

is an $n$-order polynomial with leading coefficient 1 having $n+1$ absolute extrema with alternating signs.

\section{Definition}

Polynomials $T_{1}(\theta)=1$ and $T_{n+1}(\theta)=2^{n-1} \tilde{T}_{n+1}, n \geq 1$ are called Chebyshev polynomials.

\section{Corollary}

$\overline{\text { Since } \omega_{n}(\theta)}$ is an $n$-order polynomial with leading coefficient 1 , choosing $\left\{\theta_{j}\right\}_{j=1}^{n}$ as the set of zeros of $T_{n+1}(\theta)$ scaled from domain $[-1,1]$ to $[a, b]$ leads to minimal $\left|E_{n}(\theta)\right|_{C[a, b]}$, moreover an upper estimation can be given by

$$
\left|E_{n}(\theta)\right|_{C[a, b]} \leq \frac{\max _{\theta^{*} \in[a, b]}\left\{\left|x^{(n)}\left(\theta^{*}\right)\right|\right\}}{n ! 2^{n-1}} .
$$

\section{Theorem}

The Chebyshev polynomial $T_{n+1}(\theta)=\cos (n \arccos (\theta))$ can be given by the recursive formula

$$
T_{j+1}(\theta)=2 T_{j}(\theta)-T_{j-1}(\theta), \quad j=2, \ldots, n ;
$$

with $T_{1}(\theta)=1$ and $T_{2}(\theta)=\theta$.

\section{Theorem}

If $x(\theta)$ is absolute continuous on the domain of interpolation, then its Lagrange interpolant $\tilde{x}(\theta)$ on the zeros of the Chebyshev nodes converges uniformly to $x(\theta)$ in the $C[a, b]$ norm as $n \rightarrow \infty$. 


\section{References}

[1] Åström KJ, Murray RM. Feedback Systems. Princeton University Press: New Jersey, 2008.

[2] Habib G, Rega G, Stepan G. Delayed digital position control of a single-DoF system and the nonlinear behavior of the act-and-wait controller. Journal of Vibration and Control 2014; doi: $10.1177 / 1077546314533583$.

[3] Milton J, Cabrera JL, Ohira T, Tajima S, Tonosaki Y, Eurich CW, Campbell SA. The timedelayed inverted pendulum: Implications for human balance control. Chaos 2009; 19(026110):112 .

[4] Suzuki Y, Nomura T, Casadio M, Morasso P. Intermittent control with ankle, hip, and mixed strategies during quiet standing: A theoretical proposal based on a double inverted pendulum model. Journal of Theoretical Biology 2012; 310:55-79.

[5] Insperger T, Milton J. Sensory uncertainty and stick balancing at the fingertip. Biological Cybernetics 2014; 108:85-101.

[6] Orosz G, Wilson RE, Szalai R, Stepan G. Exciting traffic jams: nonlinear phenomena behind traffic jam formation on highways. Physical Review E 2009; 80(4)(046205):1-12.

[7] Orosz G, Wilson RE, Stepan G. Traffic jams: dynamics and control. Philosophical Transactions of the Royal Society A 2010; 368:4455-4479.

[8] Takacs D, Stepan G. Contact patch memory of tyres leading to lateral vibrations of four-wheeled vehicles. Philosophical Transactions of the Royal society A 2013; 371(20120427):1-12.

[9] Stepan G. Retarded dynamical systems. Longman: Horlow, 1989.

[10] Altintas Y. Manufacturing Automation. Cambridge University Press: New York, 2012.

[11] Stepan G, Munoa J, Insperger T, Surico M, Bachrathy D, Dombovari Z. Cylindrical milling tools: Comparative real case study for process stability. CIRP Annals Manufacturing Technology 2014; 63:385-388.

[12] Breda D, Maset S, Vermiglio R. Pseudospectral differencing methods for characteristic roots of delay differential equations. SIAM Journal on Scientific Computing 2005; 27(2):482-495.

[13] Butcher EA, Bobrenkov OA. On the Chebyshev spectral continuous time approximation for constant and periodic delay differential equations. Communications in Nonlinear Science and Numerical Simulation 2011; 16:1541-1554.

[14] Vyasarayani CP, Subhash S, Kalmar-Nagy T. Spectral approximations for characteristic roots of delay differential equations. International Journal of Dynamics and Control 2014; 2:126-132.

[15] Khasawneh FA, Mann BP. A spectral element approach for the stability of delay systems. International Journal for Numerical Methods in Engineering 2011; 87:566-592.

[16] Hale JK, Lunel SMV. Introduction to functional differential equations. Springer-Verlag: New York, 1993.

[17] Diekmann O, van Oils SA, Lunel SMV, Walther HO. Delay Equations: Functional-, Complex-, and Nonlinear Analysis. Springer-Verlag: New York, 1995.

[18] Wahi P, Chatterjee A. Galerkin projections for delay differential equations. Journal of Dynamic Systems, Measurement, and Control 2005; 127:80-87.

[19] Insperger T, Stepan G. Semi-discretization for time-delay systems. Springer: New York, 2011. 
[20] Wahi P, Chatterjee A. Self-interrupted regenerative metal cutting in turning. International Journal of Non-Linear Mechanics 2008; 43:111-123.

[21] Lehotzky D, Insperger T, Stepan G. State-dependent, non-smooth model of chatter vibrations in turning. Proceedings of the ASME IDETC/CIE Conference, Boston, USA, DETC2015-46748, ASME, 2015; 1-8.

[22] Lanczos C. Applied Analysis. Prentice Hall: New Jersey, 1956.

[23] Gottlieb D, Orszag SA. Numerical analysis of spectral methods. SIAM: Philadelphia, 1977.

[24] Berrut JP, Trefethen FL. Barycentric Lagrange interpolation. SIAM Review 2004; 46(3):501-517.

[25] Michiels W, Niculescu SI. Stability and Stabilization of Time-Delay Systems. SIAM: Philadelphia, 2007.

[26] Sun JQ. A method of continuous time approximation of delayed dynamical systems. Communications in Nonlinear Science and Numerical Simulation 2009; 14:998-1007.

[27] Khasawneh FA, Mann BP. Stability of delay integro-differential equations using a spectral element method. Mathematical and Computer Modelling 2011; 54:2493-2503.

[28] Khasawneh FA, Mann BP. A spectral element approach for the stability analysis of time-periodic delay equations with multiple delays. Communications in Nonlinear Science and Numerical Simulation 2013; 18:2129-2141.

[29] Lehotzky D, Insperger T, Stepan G. Extension of the spectral element method for stability analysis of time-periodic delay-differential equations with multiple and distributed delays. Communications in Nonlinear Science and Numerical Simulation 2016; 35:177-189.

[30] Bobrenkov OA, Nazari M, Butcher EA. Response and stability analysis of periodic delayed systems with discontinuous distributed delay. Journal of Computational and Nonlinear Dynamics 2012; 7(031010):1-12.

[31] Zatarain M, Dombovari Z. Stability analysis of milling with irregular pitch tools by the implicit subspace iteration method. International Journal of Dynamics and Control 2014; 2:26-34.

[32] Breda D, Maset S, Vermiglio R. An adaptive algorithm for efficient computation of level curves of surfaces. Numerical Algorithms 2009; 52:605-628.

[33] Bachrathy D, Stepan G. Bisection method in higher dimensions and the efficiency number. Periodica Polytechnica Mechanical Engineering 2012; 56(2):81-86.

[34] Michiels W, Engelborghs K, Vansevenant P, Roose D. Continuous pole placement for delay equations. Automatica 2002; 38:747-761.

[35] Breda D, Maset S, Vermiglio R. Stability of Linear Delay Differential Equations. Springer: New York, 2015.

[36] Ito K, Teglas R. Legendre-tau approximations for functional differential equations. SIAM Journal of Control and Optimization 1986; 24(4):737-759.

[37] Breda D, Maset S, Vermiglio R. Approximation of eigenvalues of evolution operators for linear retarded functional differential equations. SIAM Journal on Numerical Analysis 2009; 50(3):14561483 .

[38] Insperger T, Stepan G. Stability chart for the delayed Mathieu equation. Proceedings of the Royal Society London A 2002; 458:1989-1998. 